Review

\title{
A Survey of Recent Synthetic Applications of 2,3-Dideoxy-Hex-2-enopyranosides
}

\author{
Ana M. Gomez *, Fernando Lobo, Silvia Miranda and J. Cristobal Lopez * \\ Instituto de Química Orgánica General (IQOG-CSIC), Juan de la Cierva 3, 28006 Madrid, Spain; \\ E-Mails: fernando.lobo@iqog.csic.es (F.L.); s.miranda@csic.es (S.M.) \\ * Authors to whom correspondence should be addressed; E-Mails: ana.gomez@csic.es (A.M.G.); \\ jc.lopez@csic.es (J.C.L.); Tel.: +34-91-258-7616 (J.C.L.); \\ Fax: +34-91-564-4853 (A.M.G. \& J.C.L.).
}

Academic Editors: Vito Ferro and Trinidad Velasco-Torrijos

Received: 16 April 2015 / Accepted: 5 May 2015 / Published: 8 May 2015

\begin{abstract}
Unsaturated carbohydrate derivatives are useful intermediates in synthetic transformations leading to a variety of compounds. The aim of this review is to highlight the rich chemistry of $\Delta-2,3$ unsaturated pyranosides, emphasizing the variety of transformations that have been carried out in these substrates during the last decade.
\end{abstract}

Keywords: hex-2,3-enopyranosides; cycloaddition; glycosylation; epoxidation; osmylation; Ferrier rearrangement; de novo synthesis

\section{Introduction}

Hex-2-enopyranoses, e.g., 3, also known as pseudoglycals, have provided fertile ground for synthetic and mechanistic developments in carbohydrate chemistry during the last decades [1,2]. The first report of a molecule belonging to this category was made by Fischer [3], although it was not until a decade later that its correct structure could be established by Bergmann [4]. However, the process $\mathbf{1} \rightarrow \mathbf{3}$ (Scheme 1), which made hex-2-enopyranosides broadly recognized synthetic intermediates, was only rendered available on a preparative scale in 1969 by Ferrier and Prasad [5]. This reaction has come to be known as the Ferrier I rearrangement, and the cationic intermediate 2 has since played a relevant role in many carbohydrate transformations [6,7]. From the outset, hex-2-enopyranosides have been employed in a plethora of synthetic endeavors [8-10]. Excellent 
coverage of the chemistry and synthetic applications of hex-2-enopyranosides has appeared regularly in the yearly issues of Carbohydrate Chemistry, Specialist Periodical Reports, until 2003 [11]. The vast contribution to the chemistry of hex-2-eno-pyranosides developed in the Fraser-Reid group, covering more than 20 years of research in the area, has recently been reviewed [12].

The aim of this review is to highlight synthetic transformations on 2,3-dideoxy-hex-2-eno-pyranosides reported during the last decade, 2003-2014.

\section{Synthetic Routes to Hex-2-enopyranosides}

The most widely used method for the preparation of 2,3-unsaturated hex-2-enopyranosides involves the Ferrier reaction, applied to glycal derivatives. Early studies on the Ferrier rearrangement made use of simple Lewis acids, e.g., $\mathrm{BF}_{3} \cdot \mathrm{Et}_{2} \mathrm{O}$, as promoters [5]. Since then, considerable attention has been devoted to the investigation of alternative catalysts for this transformation. In this context, a large number of publications involving the use of a variety of metallic, non-metallic, and heterogeneous catalysts have appeared. A report dealing with the promotors and nucleophiles currently used for the Ferrier rearrangement have been recently published, and readers in search of comprehensive information on this reaction are directed to it [13].

Besides the Ferrier rearrangement, outlined in Scheme 1, additional routes to access hex-2-enopyranoses from carbohydrates have also been described. Thus, Fraser-Reid and Boctor made use of the reductive elimination of vicinal disulfonates [14] to gain access to 5 (Scheme 2) [15].



Scheme 1. Ferrier rearrangement route to hex-2-enopyranoses $\mathbf{3}$, from glucal 1.

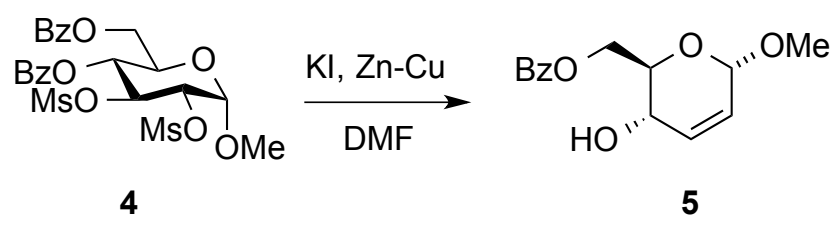

Scheme 2. Fraser-Reid and Boctor's route to hex-2-eno-pyranosides.

A more circuitous route to allylic pyranosides from non-carbohydrate sources was developed by Zamojski and Achmatowicz (Scheme 3) [16,17]. In 1971, they reported the oxidative rearrangement of 2-furanylcarbinols into highly functionalized pyranones, e.g., $6 \rightarrow 7$, to gain access to hex-2-eno-pyranosides 8 (Scheme 3). In the original Achmatowicz approach, the furfuryl carbinol is oxidized with bromine in the presence of methanol under weakly basic conditions. Many other modifications of the original Achmatowicz procedure, such as oxidation of the furan ring with 
m-CPBA [18], dimethyldioxirane [19], NBS [20,21], tert-BuOOH $\backslash \mathrm{VO}(\mathrm{OAc})_{2}$ [22], or $\mathrm{H}_{2} \mathrm{O}_{2}$-titanium silicalite [23], have also been used for this transformation. This route has the advantage that the original configuration of the alcohol moiety in the furylcarbinol is preserved and, therefore, the method is amenable to the preparation of both D- and L-series [24-26].

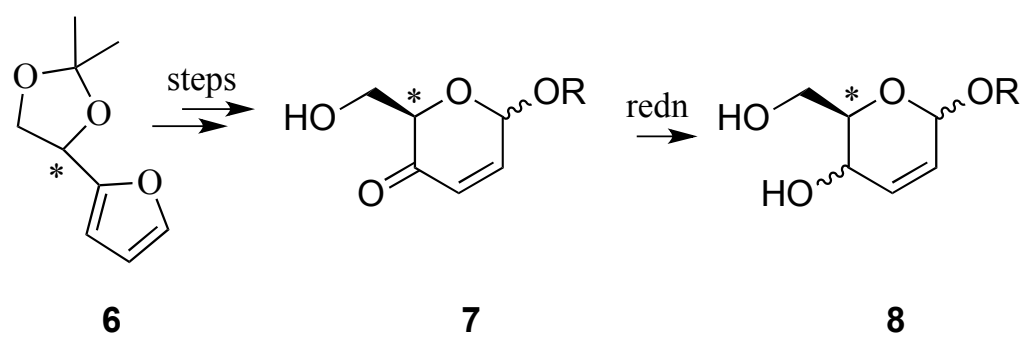

Scheme 3. Achmatowicz and Zamojski's de novo route to hex-2-enopyranosides from furylcarbinols.

The hetero Diels-Alder reaction (HDA) has been amply used in the de novo synthesis of hexoses, and in many those instances 2,3-unsaturated derivatives have been key intermediates in these protocols [27-29]. Pioneering work by Danishefsky's group had shown that hexoses could be accessed by Lewis acid-catalyzed HDA reaction of alkylated siloxy dienes with aldehydes via the intermediacy of labile 3-O-silyl-2,3-unsaturated glycoside adducts [30,31]. The hetero-Diels Alder reaction between substituted 1,4-dialkoxy-1,3-dienes and activated carbonyl compounds such as glyoxylates also provides access to hex-2-enopyranosides, e.g., 8, from non-carbohydrate sources (Scheme 4a) [32,33]. This process can be promoted simply by heating, [33], by use of high pressure [33], or by Lewis acid catalysis [32]. A HDA reaction has been used to gain access to a pseudo C-disaccharide $\mathbf{1 0}$ from a D-glucosamine diene 9 (Scheme 4b) [34]. More recently, a one-pot multicomponent approach to 3-branched-2,3-unsaturated hexopyranoses $\mathbf{1 1}$ has been devised by Botta and co-workers (Scheme 4c) [35]. The protocol, in which a monosubstituted alkyne, ethyl vinyl ether and ethyl glyoxalate were combined, involved an enyne cross-metathesis (Grubb's catalyst, 2nd generation) [36] leading a diene intermediate $(\mathbf{A})$, followed by an in situ HAD reaction.

(a)
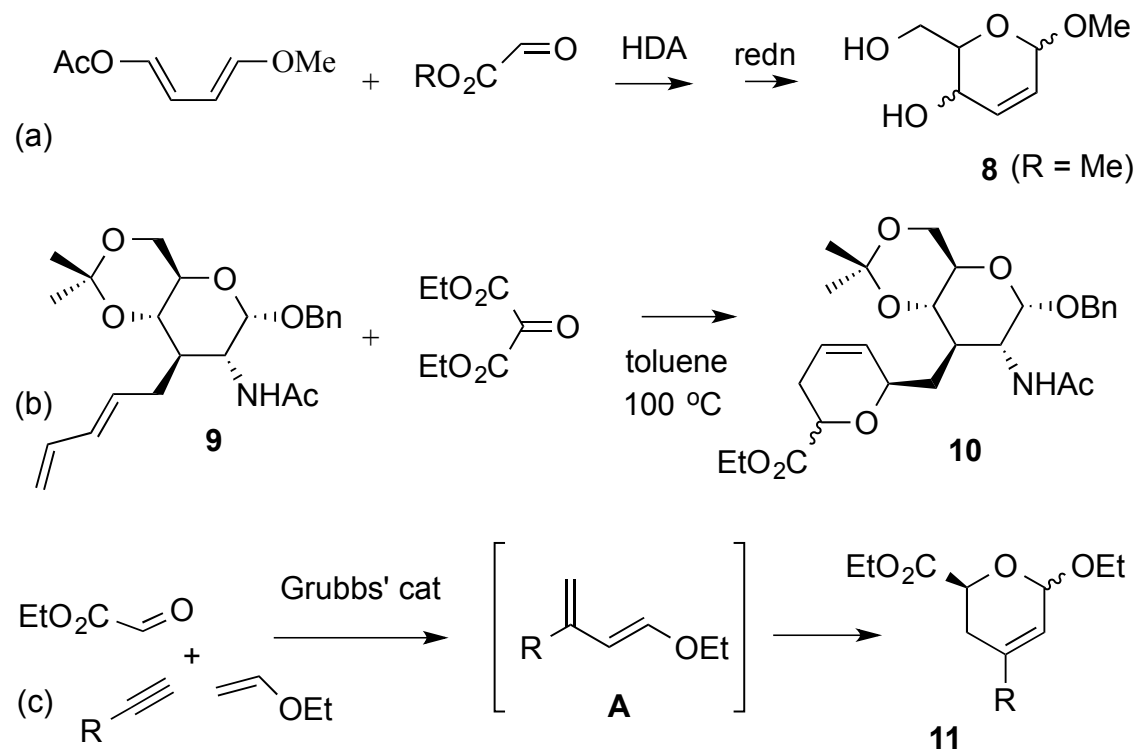

Scheme 4. Hetero Diels-Alder (HDA) routes to hex-2-eno-pyranosides. 
Ring-closure metathesis has become an important tool in organic synthesis and its application to carbohydrate chemistry [37] has included the synthesis of hex-2,3-enopyranose derivatives (Scheme 5). For example, dibenzoate 12 yielded 1-deoxy-hex-2,3-enopyranose 13 via ring-closing metathesis [38].
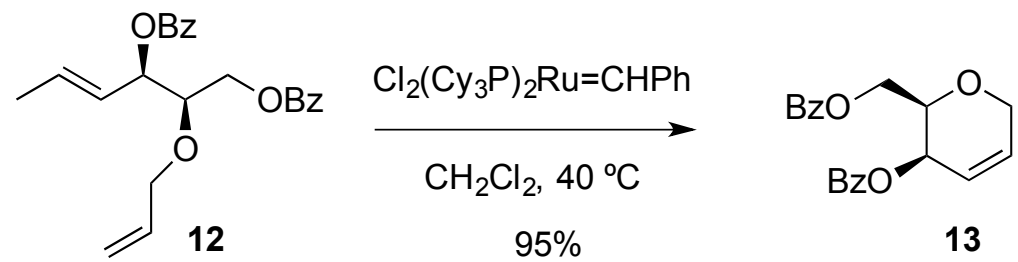

Scheme 5. Ring-closing metathesis route to hex-2-eno-pyranose derivatives.

An acid-catalyzed domino reaction has been developed by Guaragna and coworkers as a synthetic route to enantiomerically pure L-hex-2-enopyranosides (Scheme 6) [39]. Their strategy started from the three-carbon homologating agent 14, prepared in a few steps from methyl pyruvate, and a chiral building block derived from L-glyceraldehyde 15, which provides the inherent chirality at the C5 stereocenter of the final product, 18 [40]. The ring closure of the intermediate 16 was effected by a domino process triggered by DDQ in $\mathrm{CH}_{2} \mathrm{Cl}_{2} / \mathrm{MeOH}$ involving five steps: MPM protecting-group removal, oxidation of the ensuing primary alcohol, aldehyde dimethoxyacetalation, isopropylidene group cleavage, and ring closure. Finally, desulfuration of $\mathbf{1 7}$ with Raney-Ni led to 2,3-unsaturated-L-pyranoside 18.

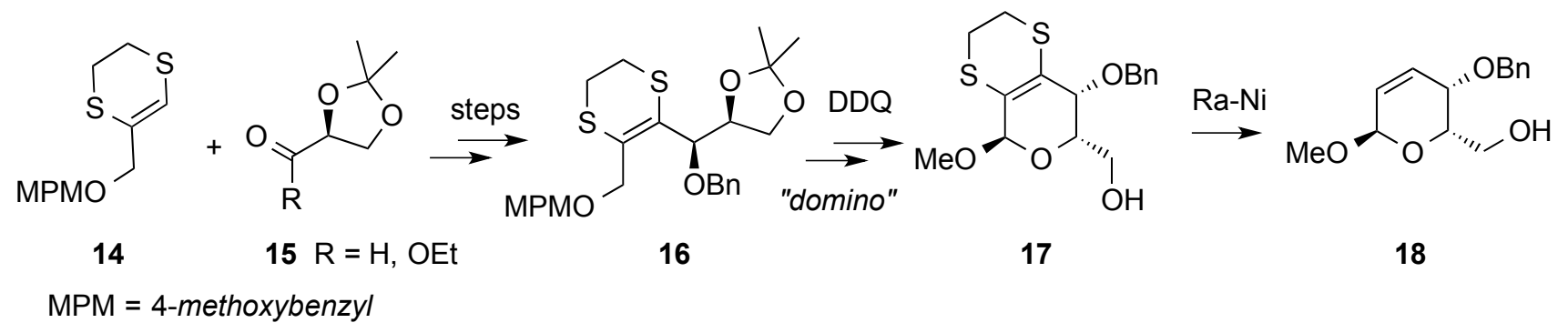

Scheme 6. Guaragna's group de novo approach to hex-2-enopyranosides.

\section{Reactions of Hex-2-enopyranosides}

One of the reasons behind the ample use of hex-2-enopyranosides in carbohydrate chemistry might lie in their rich synthetic potential. They undergo standard alkene-addition reactions including hydrogenation, hydroxylation, oxyamination, or epoxidation, often with very high if not complete stereoselectivity. Incorporation of additional functionality that polarizes the alkene group, such as nitro or sulphonyl substituents, makes Michael-like additions possible, which take place with regiospecific introduction of nucleophiles. Hex-2-enopyranosides are also ideally structured to take part in sigmatropic rearrangements, the most straightforward of which involve compounds with allylic ester groups. Furthermore, the $\Delta^{2,3}$ insaturation in hex-2-enopyranosides confers a higher reactivity to both the anomeric (C-1) acetal and the C-4 hydroxyl group, opening new avenues for nucleophilic functionalization. Oxidative transformations are also of synthetic value since they might lead to unsaturated enones, unsaturated lactones, or to 6-formyl derivatives, depending on the conditions employed. 


\subsection{Addition Reactions}

Hydrogenation reactions of 2,3-enopyranosides have generated interest as a tool for delivering deoxy sugars which are present in biologically intriguing compounds $[41,42]$. For example, it has been shown that in aminoglycosides, the removal of hydroxyl goups imparts in vitro stability by lessening the abilities of naturally occurring glycosidase enzymes to degrade the structure [43]. In this context, Zhang et al. developed a divergent strategy for constructing uncommon L-sugars with 4-substitution. They employed 2,3-eno-pyranosides 19 and $\mathbf{2 0}$ and a combination of typical palladium on carbon hydrogenation and Mitsunobu reactions involving the use of diphenylphosphorylazide (DPPA) (Scheme 7) [44].

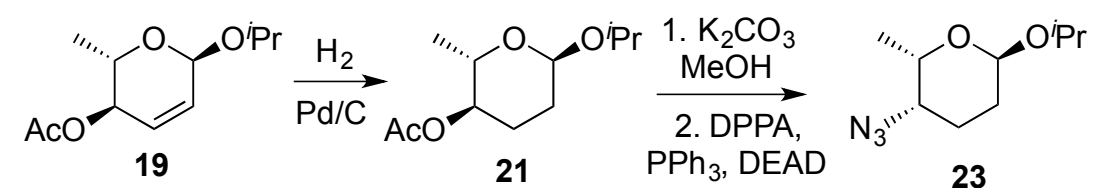

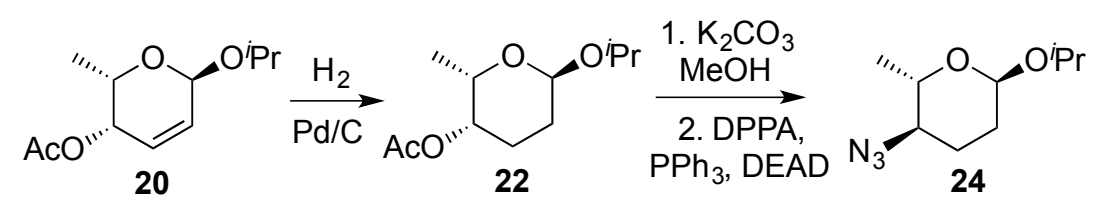

Scheme 7. Zhang's synthesis of 4-substituted uncommon-sugars.

O'Doherty's goup proposed a diimide reduction as an alternative to the direct hydrogenation reaction of 2,3-enopyranosides where partial hydrogenolysis could compete. The method was applied to allyl alcohol 25 that upon standard hydrogenation conditions produced a significant amount of the hydrogenolysis product 27 (Scheme 8). Thus, by exposing allylic alcohol 25 to an excess of $o$-nitrobenzenesulfonyl hydrazide (NBSH) and $\mathrm{Et}_{3} \mathrm{~N}$, an excellent yield of the desired pivalate $\mathbf{2 6}$ could be obtained [45].



Scheme 8. O'Doherty's diimide reduction of 2,3-enopyranosides.

Cis-hydroxylation of the double bond in hex-2,3-enopyranosides under common conditions $\left(\mathrm{OsO}_{4}, \mathrm{H}_{2} \mathrm{O}_{2}\right.$ or $\left.\mathrm{RuCl}_{3} / \mathrm{NaIO}_{4}\right)$ normally occurs from the sterically more accesible face of the sugar ring in a process that is very often stereospecific. For example, the dihydroxylation reaction of 2,3-dideoxy- $\alpha$-D-erythro hex-2-enopyranoside $\mathbf{2 8}$, where both the anomeric substituent and the 4-substituent are located below the ring, occurs exclusively from the upper face of the molecule, resulting in formation of $\alpha$-D-mannopyranoside 29 (Scheme 9a) [26]. However, osmylation of 
$\beta$-D-erythro-2-enopyranoside 30, where the C-1 and C-4 substituents are disposed in opposite faces of the pyranose, led exclusively to $\beta$-D-allopyranoside 31, with the osmium approach taking place anti- to the anomeric substituent (Scheme 9b) [46]. Similarly, dihydroxylation of galactal derivative 32 occurred mostly from the $\beta$-face opposite to the anomeric substituent leading to "talo"-derivative $\mathbf{3 3}$, although some "gulo" derivative 34 was also obtained (Scheme 9c) [47]. On the other hand, exposure of allylic alcohol 35 to $\mathrm{OsO}_{4} / \mathrm{NMO}$ in $t-\mathrm{BuOH} / \mathrm{H}_{2} \mathrm{O}$ afforded gulose isomer 36 in $80 \%$ yield, whereas the protected talose isomer $\mathbf{3 7}$ was selectively produced upon treatment of $\mathbf{3 5}$ with the TMEDA adduct of $\mathrm{OsO}_{4}$ (Scheme 9d) [26].

(a)

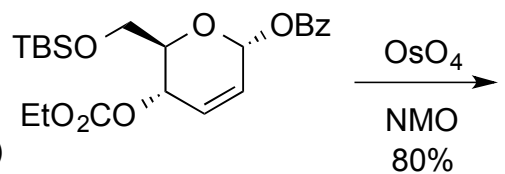

28

(b)

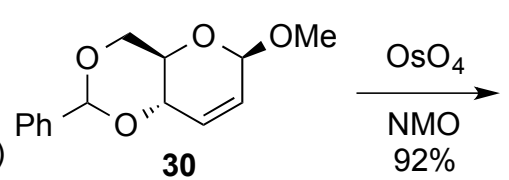<smiles>CCOC[C@H]1O[C@H](OC(=O)OCC)[C@H](O)[C@H](O)[C@H]1OC(=O)OCC</smiles>

29 "manno"<smiles>CO[C@H]1O[C@@H]2COC(c3ccccc3)O[C@H]2C[C@H]1O</smiles>

31 "allo"

(c)<smiles></smiles>

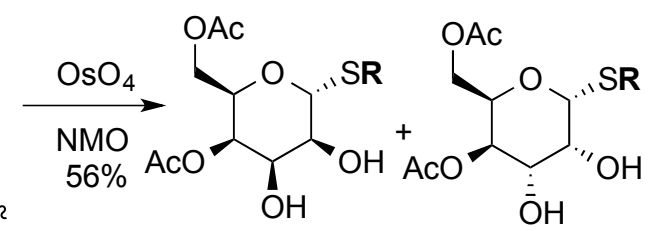

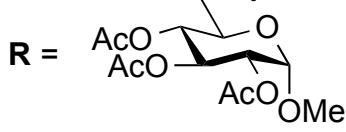

33 "talo" (1:0.4) 34 "gulo"

(d)

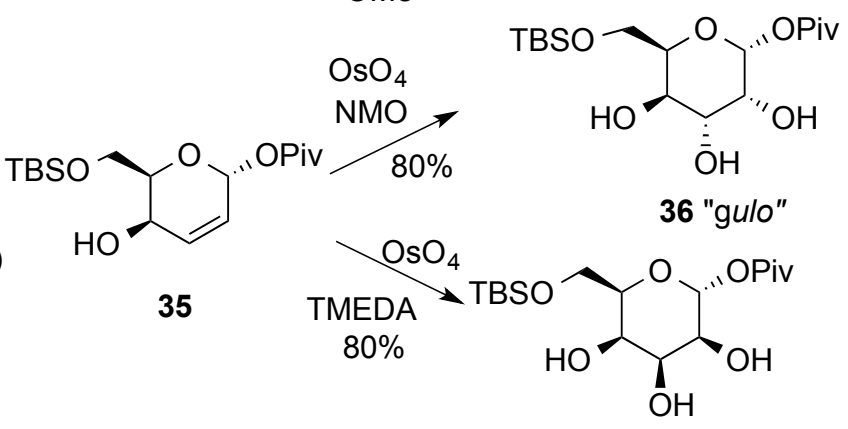

37 "talo"

Scheme 9. Cis-dihydroxylation of hex-2,3-enopyranosides by $\mathrm{OsO}_{4}$.

This methodology has been used by O'Doherty and coworkers in a highly efficient de novo route to various oligosaccharide motifs containing both D- and L-sugars [48]. For example, osmium-catalyzed dihydroxylation of tri-2,3-enopyranoside derivative $\mathbf{3 8}$ afforded the 1,4-linked $\alpha$-rhamno-pyranose 39, while the global reduction of the double bonds with excess diimide provided 2,3-dideoxy oligosaccharide $\mathbf{4 0}$ in excellent yield (Scheme 10).

Dihydroxylation products can also be obtained by sequential epoxidation/ring-opening reactions. In these substrates, the stereochemistry of the epoxidation is highly influenced by the nature of the allylic hydroxyl groups. In general, free hydroxyl groups direct the approach of the incoming oxygen atoms to the double bond in a syn manner, whereas an anti-approach is observed when the hydroxyl groups are 
protected [49]. Ring-opening of epoxides arising from hex-2,3-enopyranosides tend to form trans-diaxial products, due to the Fürst-Platnner rule [50] and therefore this approach is complementary to the previously mentioned cis-hydroxylation. For instance, hex-2-enopyranoside $\mathbf{4 1}$ under common Upjohn conditions gave exclusively methyl L-mannopyranoside 42, whereas L-altropyranoside 44 was obtained after treatment with dimethyldioxirane and the subsequent ring opening of the 2,3-anhydro derivative 43 by acid or by base-catalyzed hydrolysis (Scheme 11) [40].

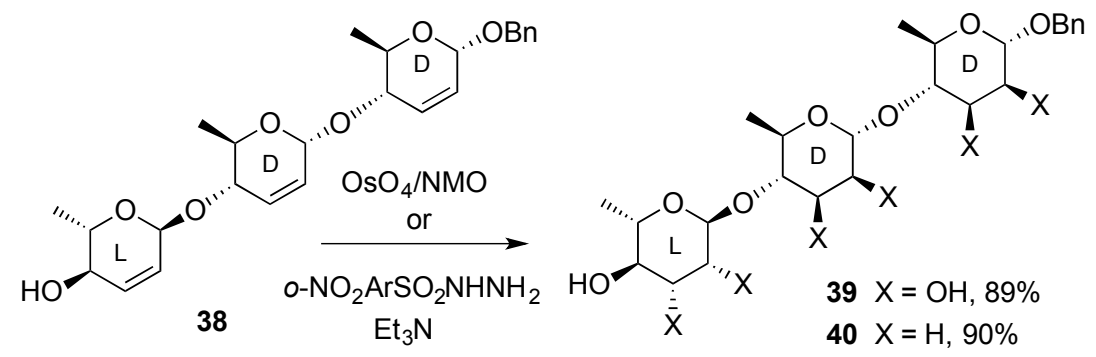

Scheme 10. O'Doherty's synthesis of 1,4-linked $\alpha$-rhamno-trisaccharides.<smiles>CC(=O)OC[C@H]1O[C@H](O[C@H](C)C(=O)OCc2ccccc2)C=C[C@H]1OCc1ccccc1</smiles>

43

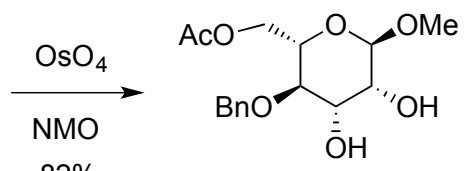

42 "manno"

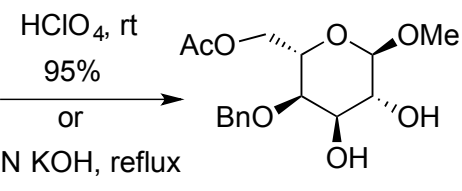

$90 \%$

Scheme 11. Alternative routes for cis- and trans-dihydroxylation of hex-2,3-enopyranoside 41.

Joly et al. [51] found that the double bond of L-sugar derivative 45 failed to react with MCPBA. However, when the reaction was performed under the conditions of Payne $\left(\mathrm{H}_{2} \mathrm{O}_{2} / \mathrm{PhCN}\right)$, a mixture of epoxides 46 and 47 was formed. The long aglycone chain is likely hindering the attack on the $\alpha$-side of the 2,3-enopyranoside and lowering the overall yield as well. The epoxides were then reductively ring-opened by $\mathrm{LiAlH}_{4}$ to form ascaroside models 48 and 49 (Scheme 12).



46

$+\quad(1: 1)$<smiles>CO[C@H]1C=C[C@@H](O)[C@@H](C)O1</smiles>

45 $30 \%$<smiles>[R]CC(C)CC(C)C</smiles>

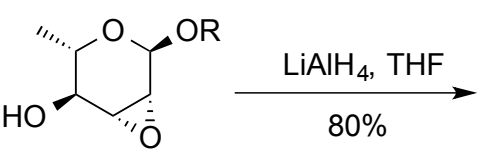

47<smiles>[R]O[C@@H]1O[C@H](C)[C@@H](O)C[C@H]1O</smiles>

48<smiles>[R]O[C@H]1O[C@H](C)[C@@H](O)C[C@H]1O</smiles>

49

Scheme 12. Synthesis of ascarosides 48 and 49. 
The incorporation of chemical functionality that polarizes the alkene on 2,3-enopyranosides makes possible Michael-like additions resulting in the regioselective introduction of nucleophiles. Several examples of Michael reactions on 3-nitro-hex-2-enopyranosides, e.g., 50, were previously reported by Sakakibara's group. In these reactions, active methylene compounds [52-54] and sterically demanding purine bases [55] reacted regio- and stereoselectively at C-2 from the side opposite to the anomeric substituent (e.g., 51 from $\alpha-50$ and $\mathbf{5 2}$ from $\beta$-50) (Scheme 13). Amines, however, produced thermodynamically more stable $\mathrm{C}-2$ equatorial products (53 and 54) irrespective of the anomeric configuration of the starting glycoside [56]. These results have been discussed in terms of electrostatic interactions [57], stereoelectronic control [57], steric hindrance [57], A-strain [58] and also hydrogen bonding [58]. Dideoxy-hex-2-en-4-ulopyranosides, on the other hand, always produced epimeric mixtures at C-2 [59-61].

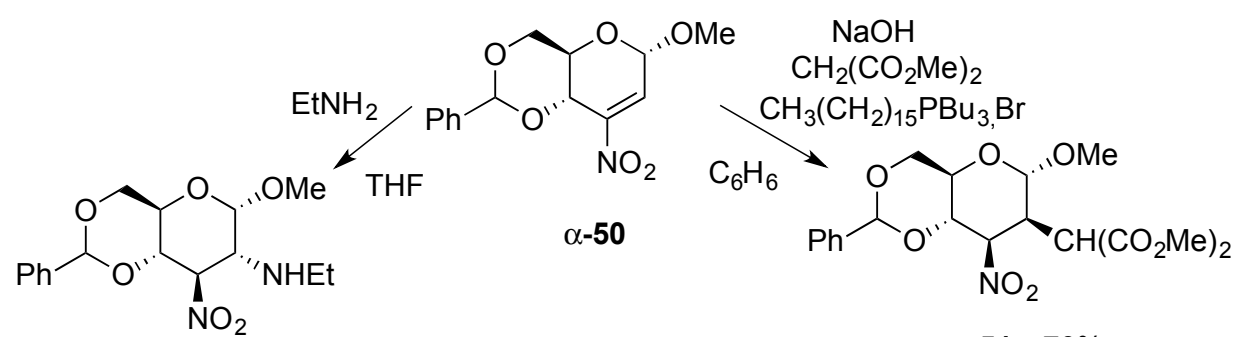

$53 \quad 95 \%$

$5170 \%$

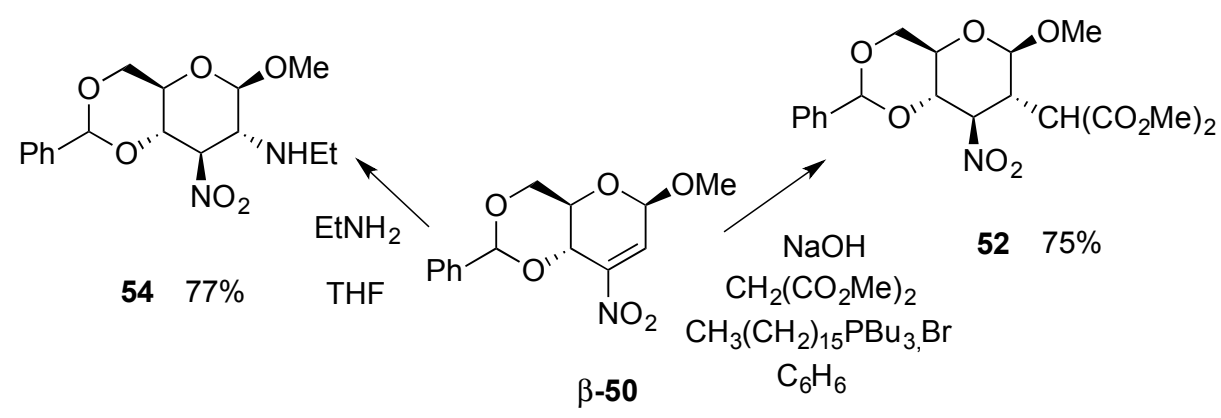

Scheme 13. Michael addition on isomeric 3-nitro-hex-2-enopyranosides 50.

More recently, Pathak and coworkers have studied the behavior of vinyl sulfone-modified hex-2-enopyranosides. Michael additions, followed by desulfonylation with $\mathrm{Na}-\mathrm{Hg}$ (6 mol-\%) of the resulting adducts [62], allowed the regio- and stereo-selective introduction of nucleophiles in 2,3-eno-pyranosides. They found a remarkable influence of the protecting groups of the hydroxyl moieties on the reaction patterns [63]. For example, although phenylmethylene-protected vinyl sulfone 55 reacts with both primary and secondary amines in a Michael-fashion, only primary amines react with the dibenzyl-protected, $O$-trityl protected or unprotected derivatives $\mathbf{5 6}, \mathbf{5 7}$ and $\mathbf{5 8}$ respectively (Figure 1).

This strategy has amply been employed by Pathak's group in the synthesis of a variety of compounds including aminosugars [64], branched-chain sugars [65], isonucleosides [62], and chiral pyrroles [66]. For example, conjugate addition of the anion generated from ethyl isocyanoacetate to vinylsulfone $\mathbf{5 5}$ afforded a pyrrole derivative 59, which by subsequent treatment with $\mathrm{POCl}_{3} / \mathrm{DMF}$ afforded chiral pyrrole 60 (Scheme 14). 
<smiles>CO[C@H]1C=C([Po])[C@H]2OC(c3ccccc3)OC[C@H]2O1</smiles>

55<smiles>[R]OC[C@H]1O[C@@H](OC)C=C([R9]OS(=O)(=O)O[R20])[C@@H]1O[R]</smiles>

$56 \mathrm{R}_{1}=\mathrm{R}_{2}=\mathrm{Bn}$

$57 \mathrm{R}_{1}=\mathrm{Tr}, \mathrm{R}_{2}=\mathrm{H}$

$58 \mathrm{R}_{1}=\mathrm{R}_{2}=\mathrm{H}$

Figure 1. Vinyl-sulfone Michael acceptors 55-58.<smiles>CCOC(=O)c1[nH]cc([C@H]2O[C@@H](c3ccccc3)OC[C@H]2O)c1C=O</smiles>

Scheme 14. Synthesis of functionalized chiral pyrrol 60 from vinylsulfone 55.

On the other hand, 2,3-unsaturated 3-arylsulfinyl pyranosides have been shown to undergo nucleophilic additions at C-2 with facial selectivities that are influenced by the nucleophile and the substituent on the sulfinyl sulphur [67]. For example, the reaction of 61a with primary amines (carbon and sulphur nucleophiles were also used) led to adduct 62a, with the addition of the nucleophile preferring an axial orientation at $\mathrm{C}-2$ and with concomitant elimination of acetic acid to form an allylic bond at $\Delta^{3,4}$. Conversely, the related reaction of $\mathbf{6 1}$ with a secondary amine led to a mixture of epimeric 2-deoxy-2-amino compounds 63a where the major product displayed a C-2 equatorial orientation. Furthermore, the influence of the $\alpha$-sulfinyl substituent on the stereochemical outcome of the reaction also became clear. Thus, reaction of sterically congested ( $p$-isopropylphenyl)vinyl sulfoxide $\mathbf{6 1 b}$ with pyrrolidine produced a C-2 $\alpha / \beta$ 7:1 epimeric mixture, whereas reaction of pyrrolidine with $p$-tolyl vinyl sulfoxide 61a produced a C-2 $\alpha / \beta$ 3:1 epimeric mixture (Scheme 15). A similar trend was also observed in the reaction of $61 \mathrm{a}$ and $\mathbf{6 1 b}$ with primary amines, leading to $62 \mathrm{a}$ and $62 \mathrm{~b}$, respectively (Scheme 15).



Scheme 15. Michael addition on 3-arylsulfinyl-hex-2-enopyranosides 61. 
Finally, hex-2-enopyranosides have shown to be popular starting materials in the preparation of biologically relevant 2,3-dideoxy-3-amino sugars in which the amino group is cis to a vicinal $(\mathrm{C} 4-\mathrm{OH})$ hydroxyl group [68]. Thus, Fraser-Reid's group introduced the iodine mediated cyclization of (C-4) allylic imidates to the $\Delta^{2,3}$ unsaturation on hex-2-enopyranosides, which directed the cis entry of the nitrogen function [69-71]. Several other functionalities such allylic carbamates or isoureas have been used since in this electrophile induced cyclization. Hydrolysis of the resulting oxazoline paves the way to the desired cis amino alcohol functionality [68]. In this context, Takahashi and co-workers have reported the synthesis of L-vancosamine, L-ristosamine, L-saccharosamine, and L-daunosamine by use of an electrophile-induced [o-iodoxybenzoic acid (IBX)] [72] cyclization of allylic carbamates [73].

\subsection{Nucleophilic Substitutions}

Reactions that allow the displacement of the C-4 allylic group on 2,3-enopyranosides also open opportunities for functionalization. Early reports were based on the nucleophilic allylic substitution with copper reagents. This possibility was limited to substrates containing acetoxy and pivaloxy, leaving groups to afford anti $\mathrm{S}_{\mathrm{N}} 2$ ' products in moderate to good yields (Scheme 16) [74-76]. In contrast, reaction of the corresponding benzothiazolyl thio ethers afforded syn $\mathrm{S}_{\mathrm{N}} 2$ ' adducts [77,78]. More recently, allylic substitution of substrates possesing the picolinoxy group have been studied and it was found that different alkyl and aryl groups could easily be installed on the pyran ring with anti $\mathrm{S}_{\mathrm{N}} 2^{\prime}$ selectivity [79].

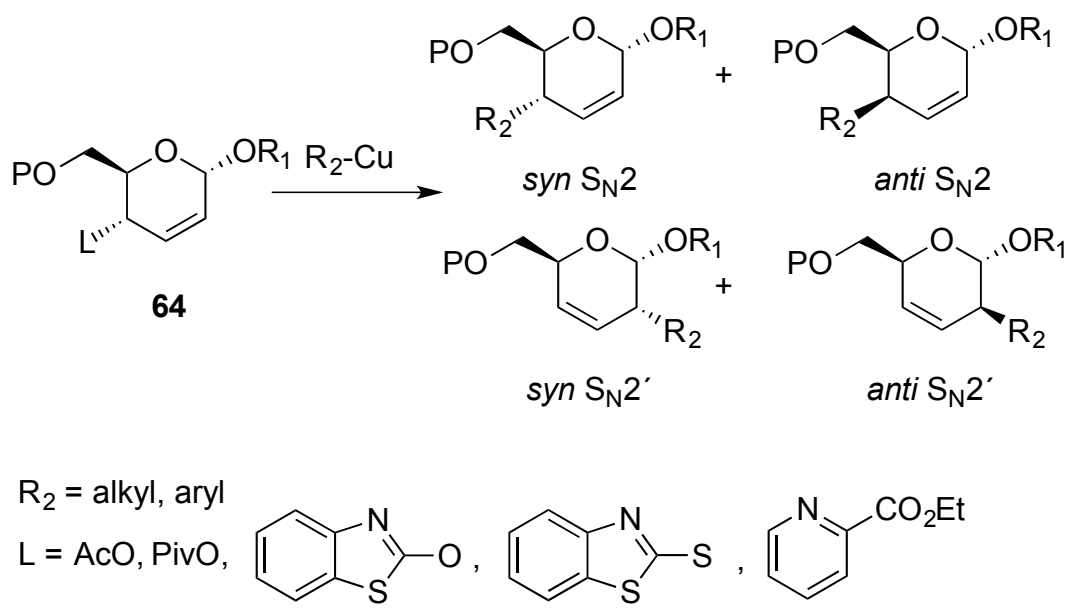

Scheme 16. Allylic substitution reaction on 2,3-enopyranoside 64 and possible regio- and stereoisomers.

Of particular relevance is the Pd-catalyzed substitution of allylic esters or carbonates by carbon and nitrogen nucleophiles [80-85]. A mechanistic picture of this process is displayed in Scheme 17. Even though one or more of these paths may become competitive, the use of more reactive allylic carbonates usually prevents the presence of any palladium(0) complex (66) in solution, and a remarkable selectivity is observed. The net overall retention in the palladium-mediated nucleophilic addition is then attributed to retention of stereochemical integrity during both generation of the $\pi$-allyl-Pd intermediate and the subsequent addition of the nucleophile. 


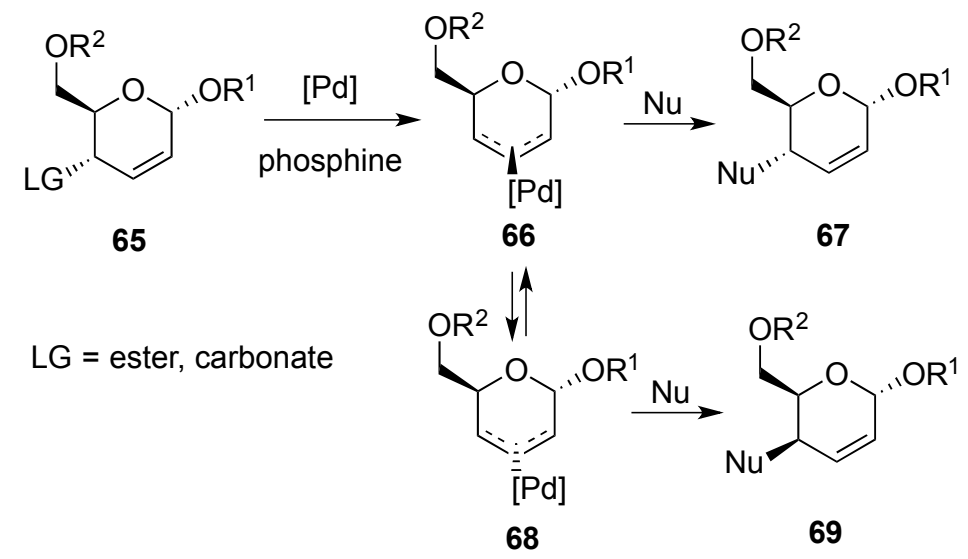

Scheme 17. Palladium-mediated allylic substitution.

This methodology has been applied to the addition of phenols [86], heterocyclic nucleophiles including uracil derivatives [87], and/or azides [88,89]. Nucleophilic substitution carried out in alkyl $\alpha$-D-erythro-hex-2- enopyranosides, e.g., 70 and 72, took place with a very high regio- and stereoselectivity to provide C-4 substituted derivatives $\mathbf{7 1}$ and 73, respectively (Scheme 18a,b). Likewise, the palladium-catalyzed reaction of $\mathbf{7 2}$ with $\mathrm{TMSN}_{3}$ led regio- and stereoselectively to 4-deoxy-4-azido derivative 74 (Scheme 18c). On the other hand, palladium-catalyzed reaction of the epimeric 2,3-dideoxy- $\alpha$-D-threo-hex-2-enopyranoside 75, with $\mathrm{TMSN}_{3}$ provided a regioisomeric mixture of 76 and 77 arising from attack at positions C-4 and C-2 of the $\pi$-allyl complex (Scheme 18d).

(a)

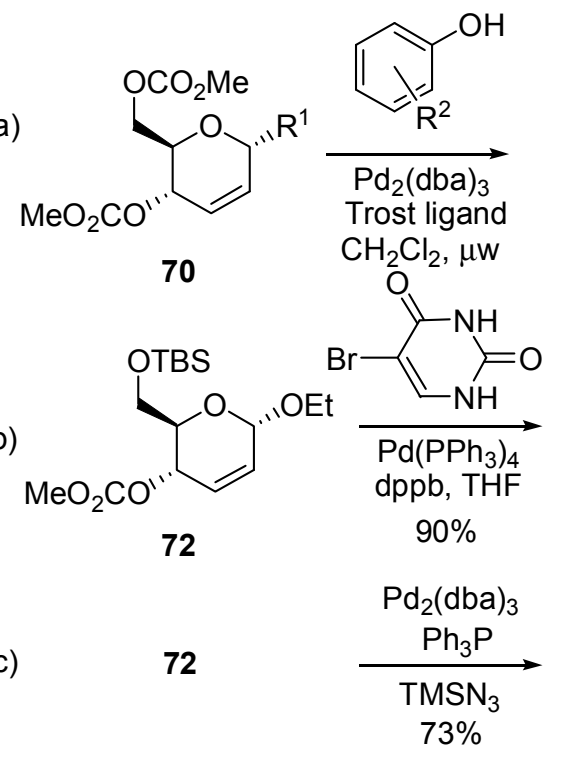<smiles>[R]c1cccc(O[C@@H]2C=C[C@@H]([R])O[C@H](COC(C)=O)[C@H]2C[O+])c1</smiles>

(c)

(d) $\mathrm{MeO}_{2} \mathrm{CO}$

75

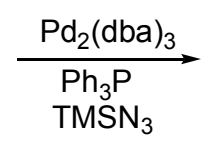

$\mathrm{TMSN}_{3}$<smiles>[Y4][C@H]1C=C[C@@H](OCC)O[C@@H]1CO[AsH3]</smiles><smiles>CCO[C@@H]1C=C[C@@H](N)[C@@H](COS(=O)(=O)c2ccccc2)O1</smiles>

$76(30 \%)$

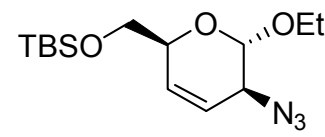

$77(46 \%)$

Scheme 18. Examples of palladium-mediated allylic substitution. 


\section{3. [3,3]-Sigmatropic Rearrangements}

Unsaturated sugar derivatives are ideally structured to take part in [3,3]-sigmatropic rearrangements that allow the construction of carbón-carbon or carbón-heteroatom bonds. For example, in 1973 Ferrier et al. showed that 4-vinyl 2,3-enopyranoside $\mathbf{7 8}$ could undergo a Claisen rearrangement upon heating at $185{ }^{\circ} \mathrm{C}$ to give the branched-chain aldehyde 79 (Scheme 19a) [90]. The reaction took place readily and in a completely stereoselective manner, as was expected for such suprafacial allyl rearrangement. However, the yield in the mercury-catalyzed preparation of the required vinyl derivative $\mathbf{7 8}$ was low (30\%). As a synthetic alternative, Krohn et al. described the reaction of the related allylic alcohol 80 with an eightfold excess of orthoacetic ester 81a in the presence of catalytic amounts of propionic acid to afford the corresponding ester 83a in good yield as one single isomer (Scheme 19b) [91]. Similarly, the Eschenmoser variant of the Claisen rearrangement allowed access to $\mathbf{8 3 b}$ ( $89 \%$ yield) from allylic alcohol $\mathbf{8 0}$ by using 1.5 equiv. of $N, N$-dimethylacetamide dimethyl diacetal 81b [91]. The C-4 epimeric allylic alcohol 84, also experienced a Claisen-Johnson rearrangement in a completely stereoselective manner leading to C-2 branched derivative $\mathbf{8 5}$, in good yield (Scheme 19c) [92].

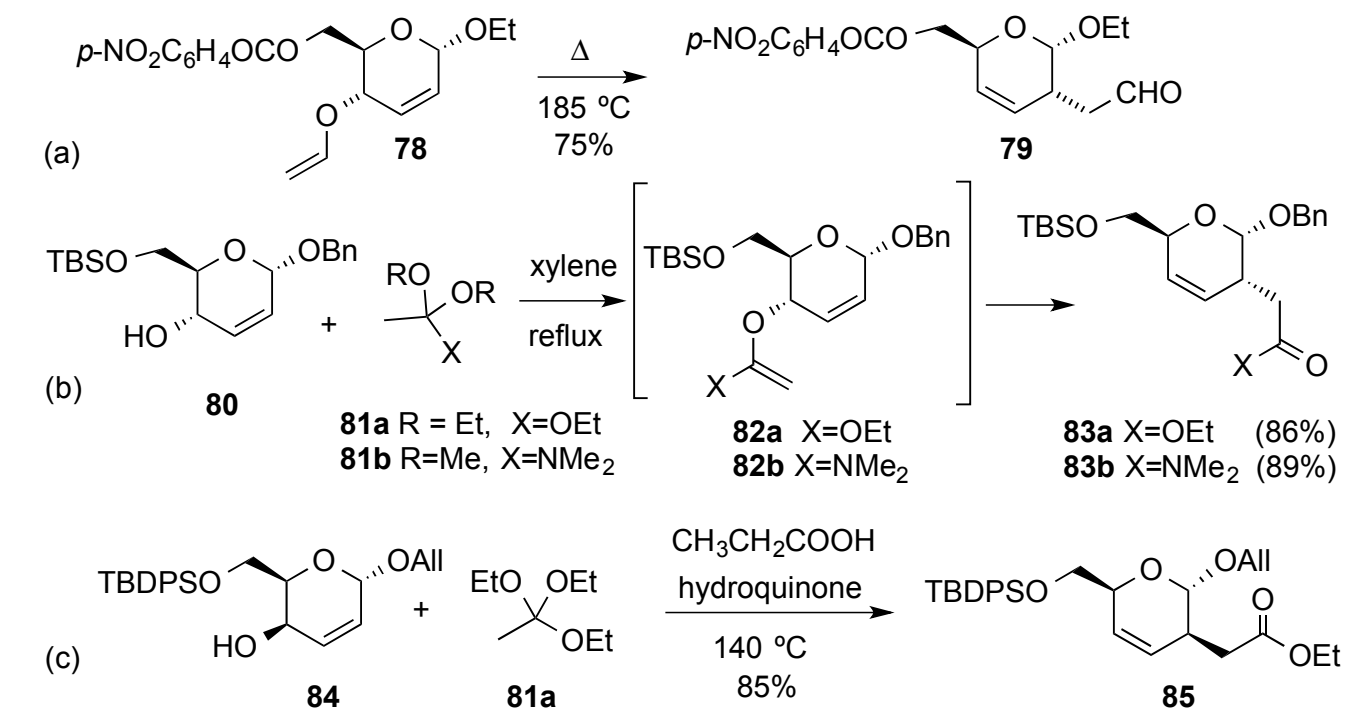

Scheme 19. Examples of Claisen rearrangements.

Porco and coworkers evaluated Eu(III)-catalyzed Claisen rearrangement of allyl phenyl ethers derived from hex-2,3-enopyranosides (Scheme 20). The reaction required microwave heating at elevated temperatures $\left(200{ }^{\circ} \mathrm{C}\right)$. Representative allyl and aryl $C$-glycosides 86a-b underwent [3,3]-sigmatropic rearrangement to provide phenols $\mathbf{8 7 a}-\mathbf{b}$ (Scheme 20). However, preliminary studies had demonstrated that this reaction depends on the aglycone substituent as alkynyl $C$-glycoside 86c did not readily undergo rearrangement, even after prolonged heating with excess $\mathrm{Eu}(\text { fod })_{3}$ [86].

Related Overman [3,3]-sigmatropic rearrangements have also been used to incorporate amine functions at $\mathrm{C}-2$ position in hex-2,3-enopyranosides. Thus, allylic trichloroacetimidate $\mathbf{8 9}$, readily obtained from $C$-allyl glycoside 88, allowed the efficient installation of a secondary amine at C-2 in compound 90 upon reflux in 1,2-dichlorobenzene in the presence of $\mathrm{K}_{2} \mathrm{CO}_{3}$ [93]. The analogous reaction with a related epimeric alcohol in allyl glycoside $\mathbf{8 4}$ required considerable experimentation, though, 
since the expected amide 92 was obtained alongside a chlorinated side-product 93 [94]. It was subsequently found that the formation of allylic chloride 93 was related to the degree of purity of trichloroacetimidate 91 used in the rearrangement. Thus, chromatographically pure imidate 91 underwent the Overman rearrangement to give the expected amide 92 in 82\% yield (Scheme 21c). However, when the rearrangement was carried out with non-purified trichloroacetimidate $\mathbf{9 1}$, and in the presence of hydroquinone as a radical scavenger, the synthetically useful chloride $\mathbf{9 3}$ could obtained as the single product in a moderate yield (Scheme 21d) [95].
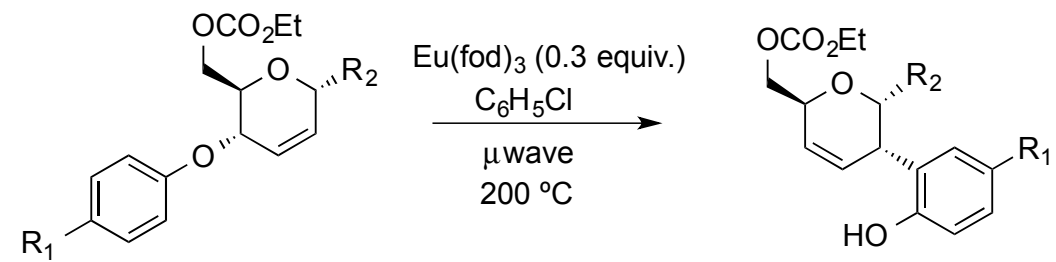

$$
\begin{array}{ll}
\text { 86a } & \mathrm{R}_{1}=\mathrm{OMe}, \mathrm{R}_{2}=\text { Allyl } \\
\text { 86b } & \mathrm{R}_{1}=\mathrm{Ph}, \mathrm{R}_{2}=p-\text { AcO-Ph } \\
\text { 86c } & \mathrm{R}_{1}=\mathrm{OMe}, \mathrm{R}_{2}=\overline{\overline{ } \mathrm{Ph}}
\end{array}
$$$$
\text { 87a } \quad \mathrm{R}_{1}=\mathrm{OMe}, \mathrm{R}_{2}=\text { Allyl } \quad(60 \%)
$$$$
\text { 87b } \quad \mathrm{R}_{1}=\mathrm{Ph}, \mathrm{R}_{2}=p-\mathrm{AcO}-\mathrm{Ph} \quad(70 \%)
$$

Scheme 20. Sigmatropic rearrangement of allyl phenyl ethers.

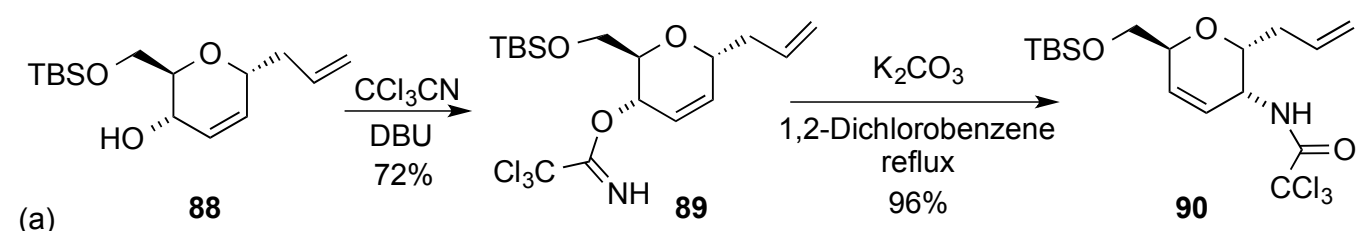

(a)

88

90

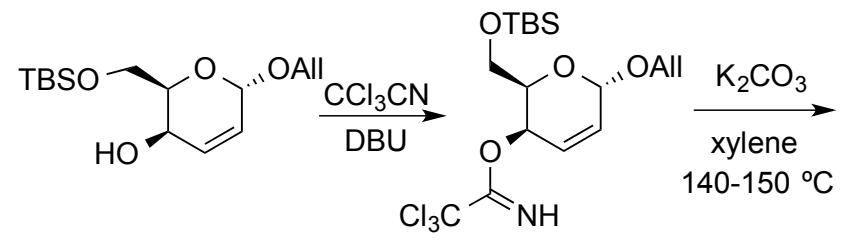



(b)

84

91

$92(23 \%)$

$93(37 \%)$

(c)

$$
\begin{array}{ccc}
\begin{array}{c}
\text { chromatographically } \\
\text { pure 91 }
\end{array} & \underset{\substack{\text { xylene } \\
140-150{ }^{\circ} \mathrm{C}}}{\stackrel{\mathrm{K}_{2} \mathrm{CO}_{3}}{\longrightarrow}} \\
\text { crude } 91 & 92(82 \%) \\
& \begin{array}{c}
\mathrm{K}_{2} \mathrm{CO}_{3} \\
\text { xylene, hydroquinone } \\
140-150{ }^{\circ} \mathrm{C}
\end{array} & \text {------- }
\end{array}
$$

$93(50 \%)$

Scheme 21. Examples of Overman rearrangements.

\subsection{Oxidative Transformations}

Oxidative transformations of hex-2,3-enopyranosides are also of synthetic value since they might lead to unsaturated enones, unsaturated lactones, or 6-formyl derivatives, depending on the conditions employed. Oxidation of diols 94 can be attained regioselectively at either $O-4$, or $O-6$, to give enones, e.g., 95 [96-101], or aldehydes, e.g., 96 [102], respectively (Scheme 22). For example, ethyl 2,3-dideoxy- $\alpha$-D-erythro-hex-2-enopyranoside $(\mathbf{9 4}, \mathrm{R}=\mathrm{Et})$ undergoes chemoselective allylic oxidation upon treatment with manganese dioxide or pyridinium dichromate to give hex-2-enopyranoside-4-ulose $\mathbf{9 5}$, 
whereas selective oxidation of the primary hydroxyl group can be effected by a modification of the Corey-Kim procedure [103], as recommended by Fraser-Reid and co-workers, leading to aldehyde 96 [104-106]. The latter compound $(\mathrm{R}=\mathrm{Et})$ was used in a stereoselective synthetic approach to $(+)$-asperlin [107].

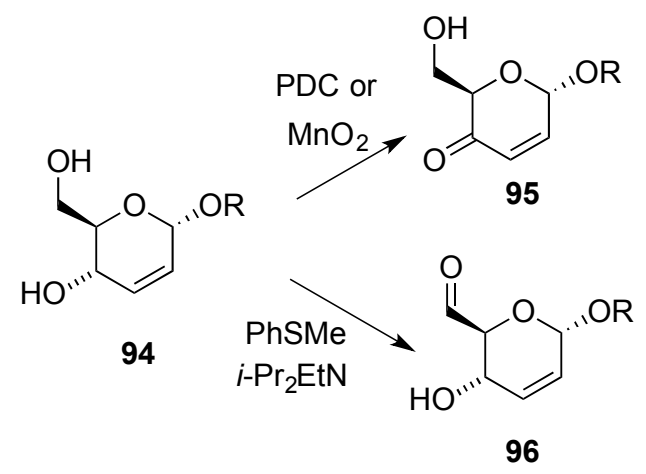

Scheme 22. Chemoselective oxidation of 2,3-dideoxy- $\alpha$-D-erythro-hex-2-eno-pyranoside 94.

On the other hand, synthetically useful 2,3-dideoxyhex-2-enono-1,5-lactones 99 can be accessed from hex-2-enopyranosides, e.g., 97, through oxidation $\left(30 \% \mathrm{H}_{2} \mathrm{O}_{2}, \mathrm{MoO}_{3}\right)$. This process, reported by Zamojski's group, involved dehydration of initially formed allylic hydroperoxides, e.g., 98 (Scheme 5) [108,109]. However, more concise routes to lactones 99 involve the direct oxidation of the corresponding glycals, and in this context the methods described by Lichtentahler's (mCPBA, $\mathrm{BF}_{3} . \mathrm{Et}_{2} \mathrm{O}$ ) and Sinaÿ's (PCC) groups are worthy of mention [110,111].

\subsection{Cycloaddition Reactions}

In order to participate in cycloaddition processes, the double bond in hex-2-eno-pyranoses has been incorporated into a variety of systems. For instance, oxidized derivatives such as enones $\mathbf{1 0 0}$ (related to 95, Scheme 22) and unsaturated $\delta$-lactones 101 (related to 99, Scheme 23) were used in Diels-Alder [9] and dipolar cycloadditions [112-115] (Figure 2). Homologated derivatives such as isomeric enals $\mathbf{1 0 2}$ and $\mathbf{1 0 3}$ also found use as dienophiles [116] and heterodienophiles [117], and finally isomeric dienes $\mathbf{1 0 4}$ and $\mathbf{1 0 5}$ were reported to undergo stereoselective Diels-Alder reactions with maleic anhydride and dimethyl acetylenedicarboxylate, among other dienophiles [116,118].

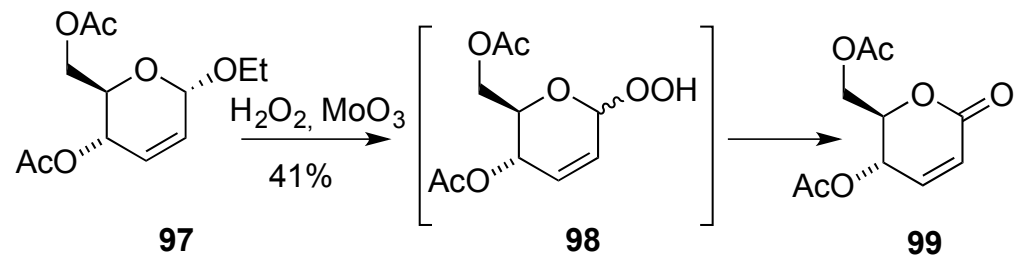

Scheme 23. Zamojski's route to unsaturated lactones from hex-2-enopyranosides.

During the last decade, Chmielewski's group has continued its investigation on 1,3-dipolar cycloaddition of nitrones to carbohydrate derived $\delta$-lactones, e.g., 101. Their research has proven useful from theoretical and practical standpoints, and some of the resulting cycloaddition adducts have been applied to the synthesis of biologically relevant iminosugars [119]. 
<smiles>[R20]CC1OC([R])C=CC1=O</smiles>

100<smiles>[R20]CC1OC([R])C=C(C)C1O[R]</smiles>

103<smiles>[R]OCC1OC(=O)C=CC1[R9]</smiles>

101<smiles>[R]CC1OC([R])C(C=C)=CC1[R]O[R]</smiles>

104<smiles>[R20]CC1OC([R])C(C=O)=CC1[R20]</smiles>

102<smiles>[R]CC1OC([R])C=C(C=C)C1O[R]</smiles>

105

Figure 2. $\Delta^{2,3}$-Unsaturated derivatives employed in cycloaddition reactions.

They have reported that the cycloaddition between aldono-1,5-lactones 106-108 and chiral five-membered cyclic nitrones $\mathbf{1 0 9}$ and 110 proceeded exclusively in the exo mode, to provide in many instances a single adduct as a result of double asymmetric induction (Figure 3) [120].<smiles>CC(=O)OC[C@H]1C=CC(=O)O[C@@H]1OC(C)=O</smiles>

106<smiles></smiles>

107<smiles></smiles>

108

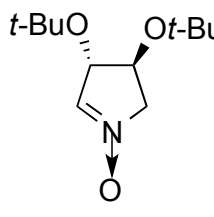

109

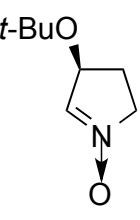

110

Figure 3. $\delta$-Unsaturated lactones and chiral nitrones.

In particular, the cycloaddition reaction between lactone 106 and nitrone 110 resulted in the completely stereoselective formation of tricyclic derivative $\mathbf{1 1 1}$ as a consequence of an exo-approach of the nitrone and the anti addition to both the acetoxymethyl- and the 4-acetoxy group of the lactone (Scheme 24). The latter was then used in the synthesis of 8-homocastanospermine 113, via key-intermediate 112, which after cleavage of the isopropylidene group and hydrogenolysis of the N-O bond underwent intramolecular alkylation of the nitrogen atom, leading ultimately to $\mathbf{1 1 3}$ [121].<smiles>CC(=O)OC[C@H]1OC(=O)C=C[C@@H]1OC(C)=O</smiles>

106

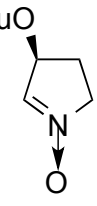

110

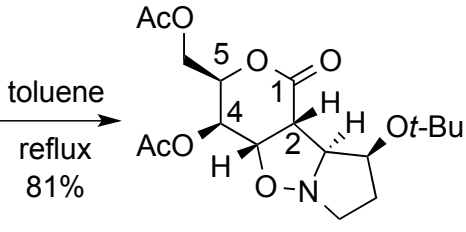

111

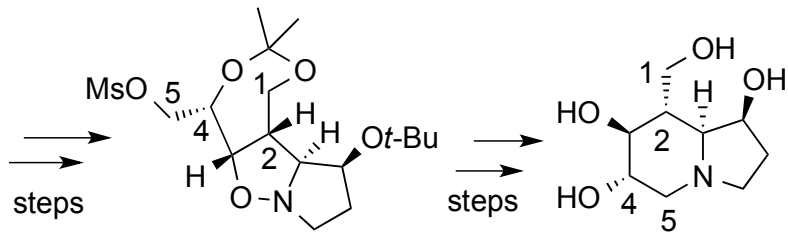

112 carbohydrate numbering

113

Scheme 24. Chmielewski's synthesis of 8-homocastanospermine 113.

Tricyclic adduct 111 was also employed in a related synthesis of a C-1 homologue of australine, 1-homoaustraline 115 (Scheme 25) [122]. Thus, chemistry related to the one mentioned-above when performed on mesylate $\mathbf{1 1 4}$ paved the way to 1-homoaustraline $\mathbf{1 1 5}$.

1,3-Dipolar cycloaddition of six-membered nitrone $\mathbf{1 1 6}$ with lactone $\mathbf{1 0 6}$ gave one single adduct, $\mathbf{1 1 7}$, as the result of the exo-anti approach to both substituents of the lactone dipolarophile (Scheme 26) [123]. The latter was next transformed through a series of synthetic steps into mesylate 117 that led to 2,3-dihydroxy-epilupinine 119. 


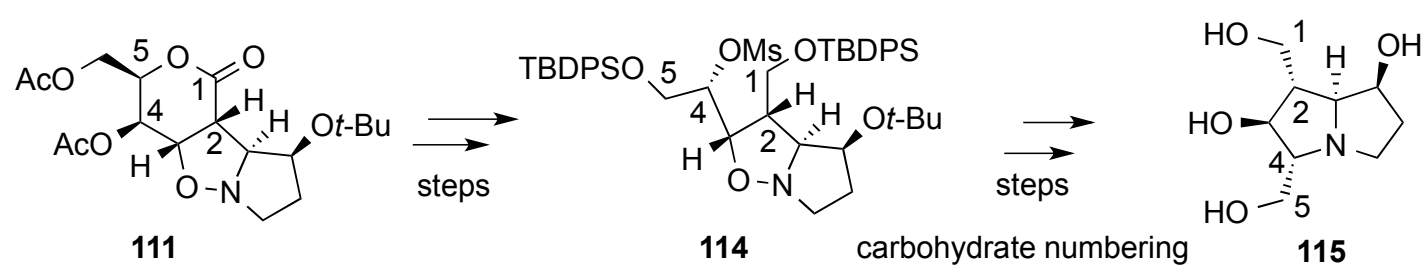

Scheme 25. Chmielewski's synthesis of 1-homoaustraline 115.

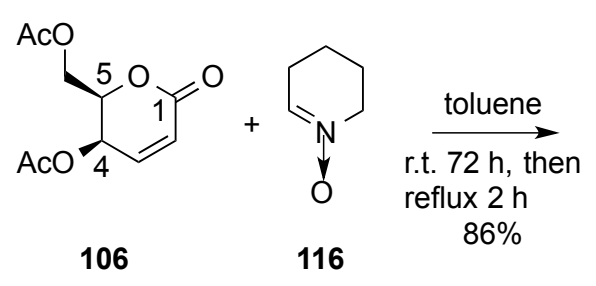

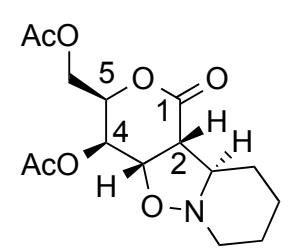

117

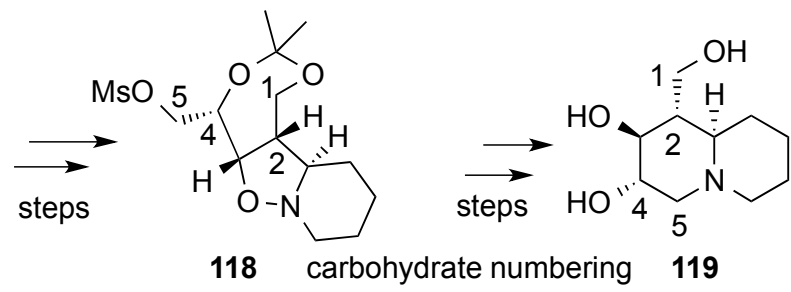

118 carbohydrate numbering 119

Scheme 26. Chmielewski’s synthesis of 1-epilupinine 119.

The syntheses described above have benefitted from two key issues: (i) the high stereoselectivity of cycloadditions of simple nitrones to threo-lactones, e.g., 106, when compared to erythro-lactones, e.g., 107, and (ii) the easy rearrangement of the $\delta$-lactone fragment in the adduct to a $\gamma$-lactone whose terminal diol could easily be cleaved.

Along this line, the cycloaddition of acyclic nitrones to carbohydrate-lactones was also studied for the preparation of iminosugars (or azasugars) [124] In this context, cycloaddition of nitrone $\mathbf{1 2 0}$ and lactone 106 produce one single adduct, 121, which was processed by rearrangement to a $\gamma$-lactone and removal of the terminal (C-6) hydroxymethyl group into mesyl derivative 122 (Scheme 27). Deprotection of the hydroxy groups in the latter caused immediate intramolecular alkylation of the nitrogen atom leading to ammonium salt 123. Finally, hydrogenolysis of $\mathbf{1 2 3}$ followed by several acetylation/deacetylation processes and hydrogenolysis of the $N$-benzyl substituent afforded (-)-isofagomine 124 [125].

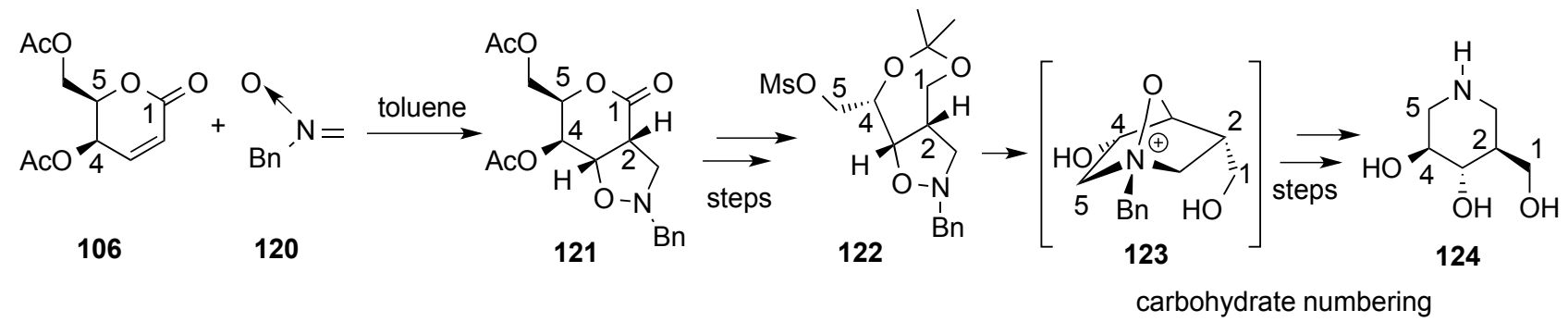

Scheme 27. Chmielewski's synthesis of (-)-isofagomine 124.

Recent work by Chmielewski's group has shown that the 1,3-dipolar cycloaddition to $\alpha, \beta$-unsaturated $\delta$-lactones is under kinetic control. Conversely, when $\gamma$-lactones are involved in the cycloaddition, the process becomes reversible and could be used to obtain the more stable, thermodynamic products. Owing to these properties along with the above-shown high stereoselectivity of their cycloaddition, $\delta$-lactones have been employed for the kinetic resolution of racemic nitrones [126].

Testero and Spanevello reported a concise synthetic route to pentanelactone $\mathbf{1 2 8}$ from $\alpha, \beta$-unsaturated aldehyde 125 (Scheme 28) [127]. The successful approach was based on two key steps. First, a completely 
stereoselective Diels-Alder cycloaddition of cyclopentadiene and enal 125 [128], with the diene approaching the dienophile from the $\beta$-face in an exo-mode of addition. Second, the ozonolysis of 126 took place via a completely regioselective cleavage leading to dialdehyde $\mathbf{1 2 7}$. The latter was then transformed in ten steps into pentalenolactone $\mathbf{1 2 8 .}$



Scheme 28. Synthetic approach to pentalenolactone 128.

Further studies by this group have addressed the issue of the fragmentation of the primary ozonide in carbohydrate-derived norbornene systems. They showed that, in participating solvents, the remote substitution is responsible for the regioselective fragmentation of the intermediate ozonide [129-131].

A multistep route to previously described enal 135 [128], has also been described (Scheme 29) [132]. The synthesis started with the oxidative cleavage of methyl- $\alpha$-D-glucopyranoside 129 leading to dialdehyde 130. Treatment of $\mathbf{1 3 0}$ with nitromethane in basic medium led to a mixture of 3-deoxy-3-nitro derivatives 131. Benzylidenation of these derivatives followed by purification of the crude reaction provided epimeric mixture of alcohols 132 that upon elimination, mediated by treatment with $\mathrm{MsCl}$ and $\mathrm{Et}_{3} \mathrm{~N}$, yielded unsaturated nitro derivative 133. Further processing of 133 via cyano derivative 134 permitted access to $\mathbf{1 3 5}$.

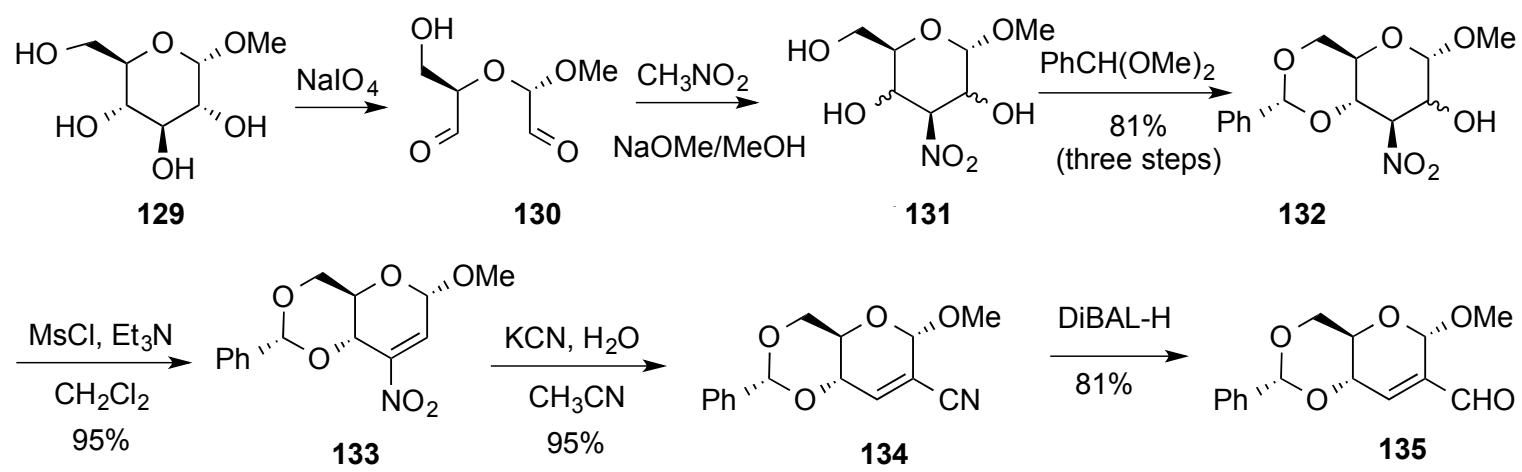

Scheme 29. Spanevello's synthetic route to enal $\mathbf{1 3 5}$.

Finally, the thermal Diels-Alder reaction between sugar-derived nitroalkene $\mathbf{1 3 3}$ and cyclopentadiene yielded a mixture of exo- and endo- adducts 136 and 137, respectively, where unlike previous examples, an $\alpha$-facial selectivity in the approach of the diene was observed (Scheme 30) [133].<smiles>CO[C@H]1C=C([N+](=O)[O-])[C@H]2O[C@H](c3ccccc3)OC[C@H]2O1</smiles>

133

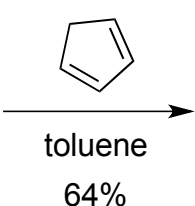

$64 \%$

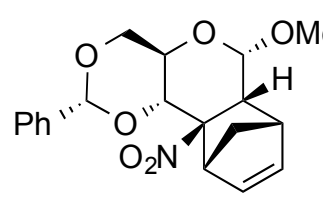

136

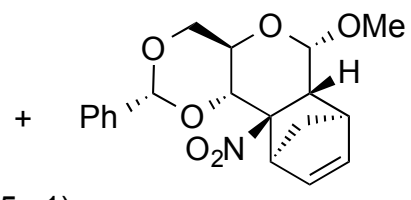

137

Scheme 30. Diels-Alder cycloaddition between nitroalkene 133 and cyclopentadiene. 


\subsection{Glycosylation Reactions}

The study of glycosylation reactions of 2,3-unsaturated hexenopyranoses has recently been addressed by mediation of either palladium or Lewis acid catalysis.

\subsubsection{Palladium Mediated Glycosylation}

Feringa and O'Doherty's groups addressed the issue of glycosylation with 2,3-unsaturated hexoses functioning as glycosyl donors [134].

Following previous studies on palladium catalyzed allylic substitution on 6-acetoxy-2H-pyran3(6H)-ones by alcohols (Scheme 31a) [135], Feringa and co-workers reported the stereoselective palladium catalyzed glycosylation of pyranones (Scheme 31b) [136]. The method proved to be particularly useful in synthesis since retention of stereochemistry at the allylic acetal moiety was observed in the newly formed glycosidic bond.

(a)

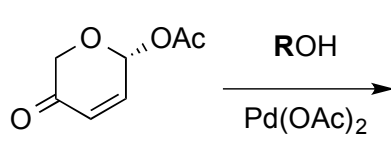

138

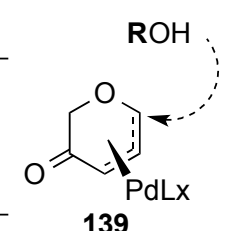

139

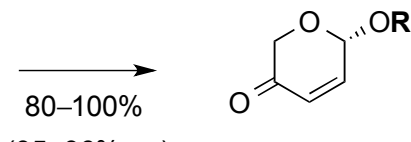

(95-96\% ee) $\quad 140$

$\mathbf{R}=\mathrm{Me}, \mathrm{Et}, i-\mathrm{Pr}$<smiles>O=C(O[C@H]1C=CC(=O)[C@@H](CO[R15](F)(F)F)O1)c1ccccc1</smiles>

(b)<smiles>COc1cccc(CO)c1</smiles>

$\mathrm{P}(\mathrm{OPh})_{3}$ $\mathrm{CH}_{2} \mathrm{Cl}_{2}$

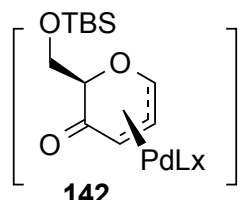

142

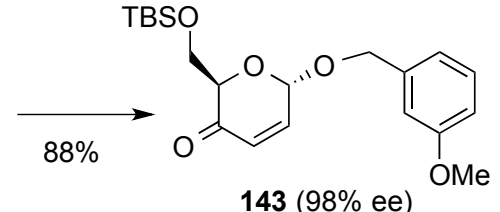

Scheme 31. Feringa and co-workers' palladium-catalyzed allylic substitution.

They next explored the feasibility of an iterative protocol based in this chemistry for saccharide synthesis. Thus, diastereoselective catalytic cis-dihydroxylation of $\mathbf{1 4 4}$ followed by acetonide formation on the ensuing diol and reduction of the ketone moiety paved the way to $\beta$-L-ribose derivative $\mathbf{1 4 5}$ (Scheme 32). This sugar was next glycosylated with (-)-138 under palladium catalysis $\left[\mathrm{Pd}_{2}(\mathrm{dba})_{3}\right.$, $\left.\mathrm{PPh}_{3}\right]$ to give disaccharide precursor 146.<smiles>COc1cccc(CO[C@@H]2C=CC(=O)CO2)c1</smiles>

144

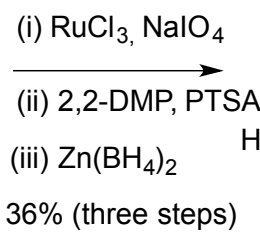

$36 \%$ (three steps)<smiles>CO[C@H]1OC[C@@H](O)C2OC(C)(C)O[C@@H]21</smiles>

145

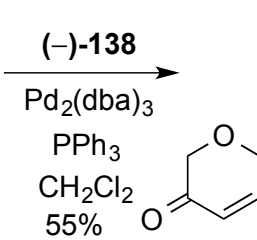

$55 \%$<smiles>COc1cccc(CO[C@@H]2OC[C@@H](O)C3OC(C)(C)OC32)c1</smiles>

146

Scheme 32. Feringa's approach to iterative saccharide synthesis.

Shortly after Feringa's findings, O'Doherty's group reported on a similar transformation [137]. They studied the behavior of $\mathrm{Pd} \pi$-allyl intermediates 149 and 150 arising from allylic alcohols 147 or unsaturated ketone 148, respectively, and found that whereas reaction of 147 failed to provide any 
unsaturated glycoside 151 (Scheme 33a), $\pi$-allyl intermediate $\mathbf{1 5 0}$ reacted with a variety of alcohols to give allylic glycosides 152 in moderate to excellent yields (Scheme 33b). O'Doherty's group ascribed these contrasting results to the higher electrophilicity of $\mathrm{Pd} \pi$-allyl intermediate $\mathbf{1 5 0}$ compared to 149. In this context, Lee and co-workers reported that the reaction of intermediates type 149, generated from glycals rather than from hex-2,3-enopyranosides, with alcohol acceptors to give $O$-glycosylation products, e.g., 151, could be carried out by activating the acceptor via zinc(II) alkoxide formation [138,139].

(a)<smiles>[R5]OC[C@H]1O[C@@H](OC([R])=O)C=C[C@H]1[R20]</smiles>

147

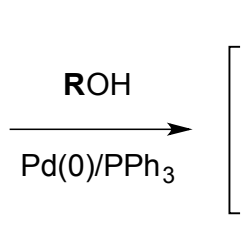$$
\text { L }
$$

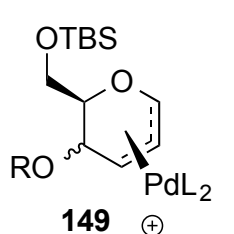

$149 \oplus$

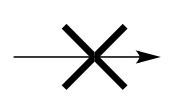<smiles>[R]C(=O)O[C@H]1C=CC(=O)[C@@H](CO[AsH3])O1</smiles>

(b)

148

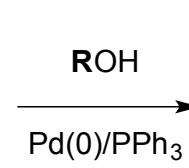

$52-89 \%$

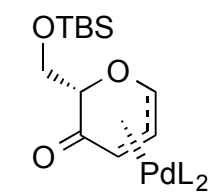

$150 \oplus$<smiles>[R]O[C@H]1C=C[C@@H]([R20])[C@@H](C[SeH])O1</smiles>

151

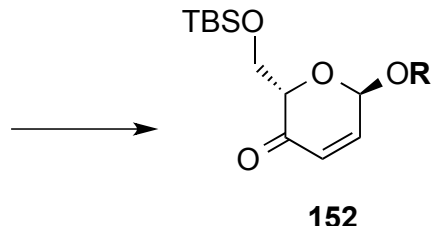

series: $\mathbf{a} \mathrm{R}^{1}=\mathrm{Ph} ; \mathbf{b} \mathrm{R}^{1}=t-\mathrm{Bu} ; \mathbf{c ~}^{1}=\mathrm{O} t-\mathrm{Bu}$

Scheme 33. O’Doherty's Pd-catalyzed glycosylation with pyranone donors 148.

Further experimentation by O'Doherty's group led to the use of tert-butyl carbonates $\mathbf{1 4 8 c}$ as the preferred glycosyl donors. Their explanation for the improved reactivity of $148 \mathrm{c}$ versus $\mathbf{1 4 8 a}, \mathbf{b}$, was that $t$ - $\mathrm{BuOH}$ and $\mathrm{CO}_{2}$, rather than carboxylic acids, were generated as leaving groups.

The Pd-catalyzed glycosylation reaction proceeded with high selectivity for both, $\alpha$ - and $\beta$-glycosylation. Thus, using either donor $148 \alpha$ or $148 \beta$ provided the corresponding glycosides $152 \alpha$ or $152 \beta$, with retention of stereochemical integrity at the anomeric center (Scheme 34a,b). The scope of the reaction was investigated with an array of alcohol nucleophiles (Scheme 34). The use of sterically hindered adamantol as a glycosyl acceptor led to moderate yields of adamantyl glycosides $(\approx 52 \%-54 \%$ ) along with tert-butyl glycoside $\mathbf{1 5 3 \beta}$ (in the glycosylation of $148 \beta$, Scheme $34 \mathrm{~b}$ ). Formation of the latter was explained by the presence of "departing" $t$-BuOH as a competing nucleophile in the reaction media. However, the use of excess glycosyl acceptors or the use of pyvaloyl rather than tert-butoxy carbonyl glycoside donors allows increased yield in the formation of adamantyl glycosides.

The starting pyranones $\mathbf{1 4 8}$ were easily accessible from furan alcohols by Achmatowicz ring-expansion [16,17] followed by stereoselective hemiacetal protection [140].

The diastereoselective palladium-catalyzed glycosylation was also used in the preparation of the pheromone daumone 154, by use of pyranone 155 as the glycosyl donor (Scheme 35) [20]. The latter was prepared in enantiomerically pure form by enantioselective Noyori reduction [141] of acylfuran 157 (Scheme 36). Thus, Noyori reduction of 157 with the enantiomeric catalyst provided furan alcohol 158 in very high enantiomeric excess (93\% yield, >96\% ee). Ring-expanded pyranone 159 was then obtained by treatment with $\mathrm{N}$-bromosuccinimide (NBS) in $\mathrm{THF} / \mathrm{H}_{2} \mathrm{O}$ ) $[16,17]$. Diastereoselective acylation of $\mathbf{1 5 9}$ was performed at low temperature with (Boc) $)_{2} \mathrm{O}$ provided pyranone donor 155. 


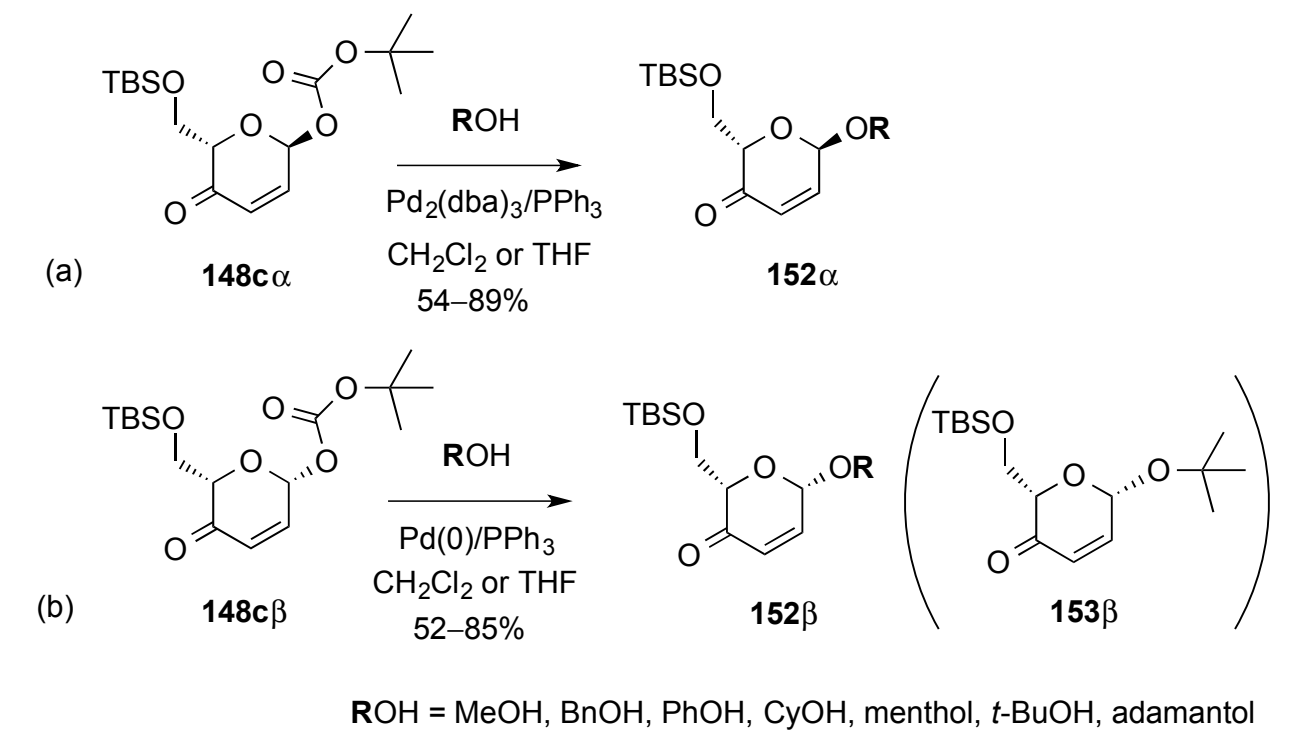

Scheme 34. Pd-catalyzed stereoselective glycosylation.

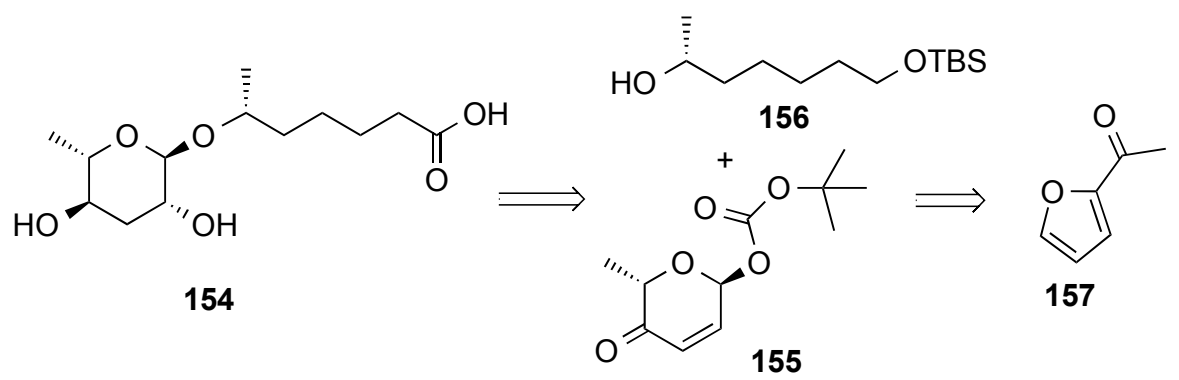

Scheme 35. O'Doherty's approach to daumone, 154.

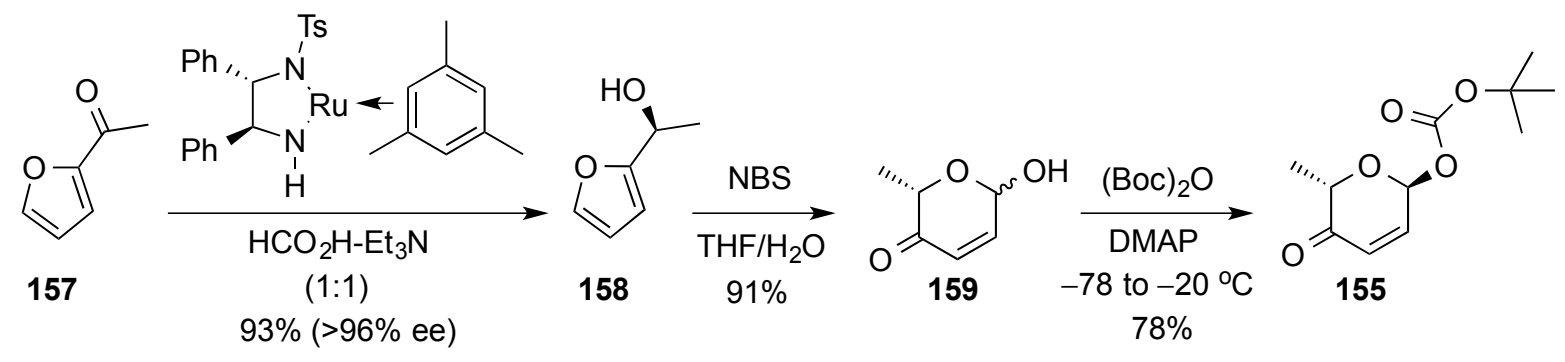

Scheme 36. Enantioselective synthesis of $\alpha$-pyranone 155.

Palladium-catalyzed glycosylation of secondary alcohol 156 with $\alpha$-pyranone 155 was carried out in $\mathrm{CH}_{2} \mathrm{Cl}_{2}$ to yield glycoside $\mathbf{1 6 0}$ as a single diastereomer (Scheme 37). Diastereoselective epoxidation of enone 160 to give epoxy-ketone 161 was then followed by a one-pot process involving ketone reduction and epoxide opening to give rhamnose derivative 162. Finally, deprotection and oxidation led to daumone $\mathbf{1 5 4}$.

O'Doherty's group has also exploited this methodology for the synthesis of oligosaccharides, in particular 1,6-linked and 1,4-linked oligosaccharides, by way of iterative glycosylations combined with diastereoselective ketone reduction and dihydroxylation processes [142]. 


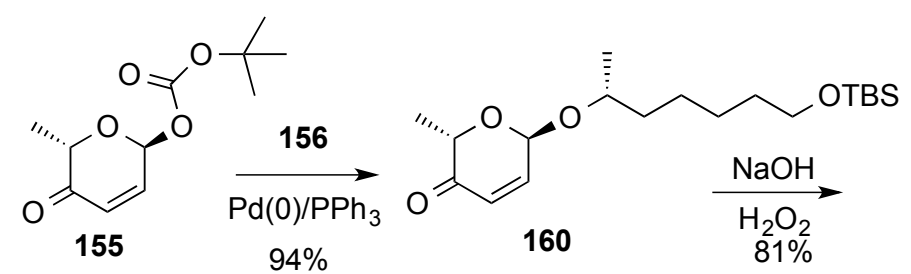<smiles>C[C@@H](CCCCC[18OH])O[C@@H]1O[C@H](C)C(=O)[C@H]2O[C@H]21</smiles>

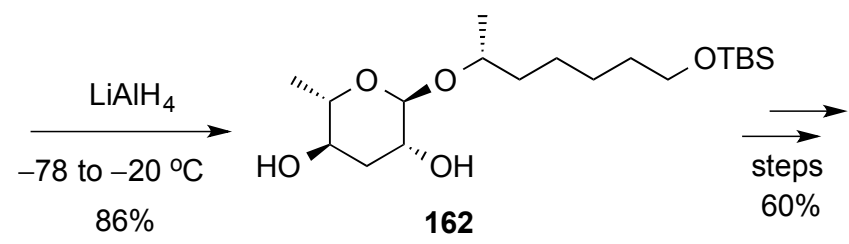<smiles>C[C@H](CCCCC(=O)O)O[C@@H]1O[C@H](C)[C@H](O)C[C@H]1O</smiles>

Scheme 37. Palladium-catalyzed glycosyl coupling and processing to daumone $\mathbf{1 5 4}$.

The iterative glycosylation protocol was also applied to the stereoselective synthesis of digitoxin 163 (Scheme 38) [143,144]. O'Doherty's retrosynthesis for digitoxin is outlined in Scheme 38 and involved the iterative, diastereoselective, palladium catalyzed glycosylation of digitoxigenin (165) with pyranone 164, which is accessible in enantiomerically pure form from acylfuran 157.

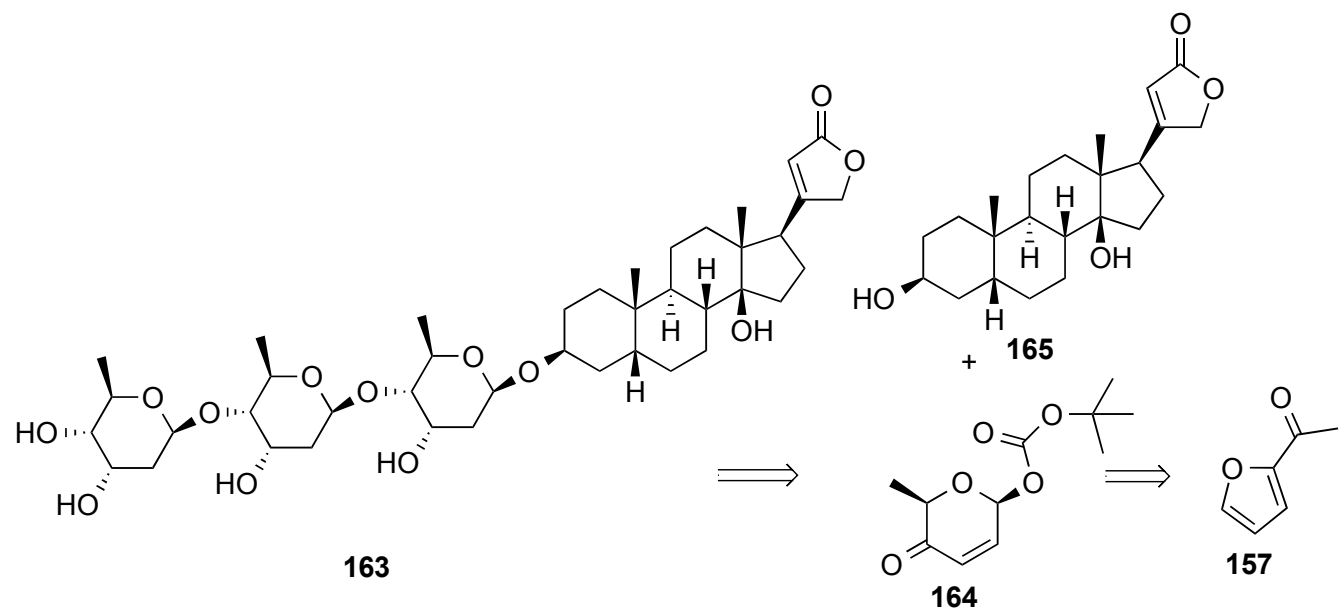

Scheme 38. O’Doherty's retrosynthesis of digitoxin 163.

The synthetic route started with an enantioselective Noyori reduction of acylfuran 157, followed by the Achmatowicz ring-expansion protocol, and stereoselective anomeric Boc-formation at high temperature to obtain pyranone $\mathbf{1 6 4}$ as the major isomer (Scheme 39). Palladium-catalyzed gycosylation of digitoxigenin (165) with 164 produced glycoside 166, which was processed to dihydroxy acetate 167. Iteration of the glycosylation/pyranose functionalization processes to the di- and trisaccharides 168 and 179, respectively, resulted in the synthesis of digitoxin, 163.

The usefulness of the protocol implemented by O'Doherty's group from achiral furan 157 via enantioselective reduction, Achmatowicz ring-expansion, and diastereoselective (iterative) palladium-catalyzed glycosylation(s) has been further demonstrated with the successful synthesis of anthrax tetrasaccharide (171) [145-147], the trisaccharide portion of landomycin A (172) [148], cleistroside-2 (173) and several members other members of the cleistroside (tri- and tetra-rhamnosides) 
family [149], as well as the total syntheses of kaempferol glycoside SL101 (174) [150], jadomycin B (175) [151,152], and vineomycinone $B_{2}$ methyl ester (176) [153] (Figure 4) [154].
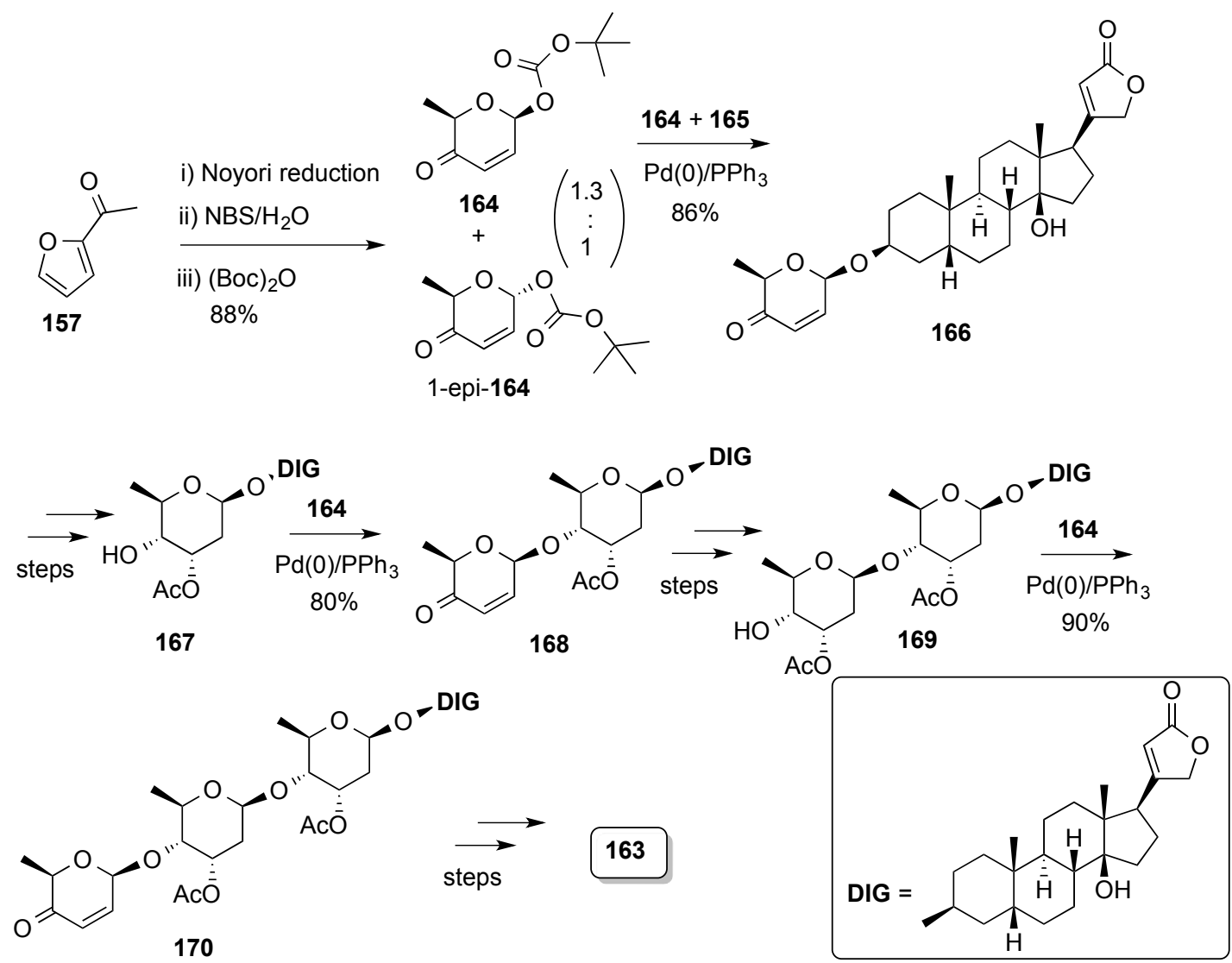

Scheme 39. O'Doherty synthesis of digitoxin (163) by iterative palladium-catalyzed glycosylations.

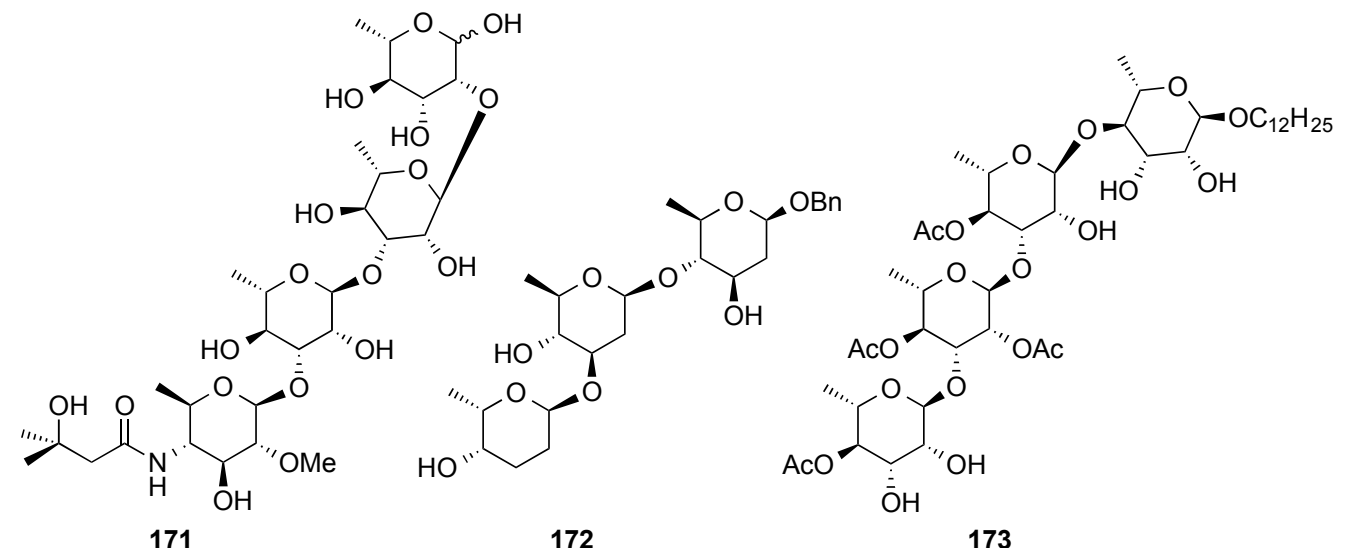<smiles>CC(=O)OC1C(C)OC(Oc2c(-c3ccc(O)cc3)c3c(O)cc(O)cc3oc2=O)C(O)C1OC(C)=O</smiles>

174

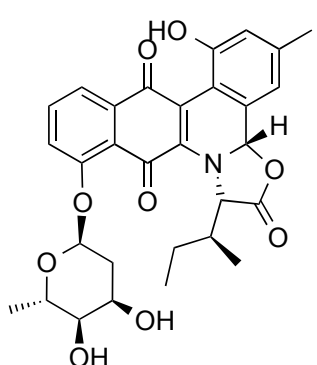

175<smiles>CC(=O)CC(C)(O)Cc1ccc2c(c1O)C(=O)c1ccc(OC3CC(O)C(C)OC3C)c(O)c1C2=O</smiles>

176

Figure 4. Natural products synthesized by O'Doherty's group. 
The synthetic potential of this protocol is enhanced by the flexibility of the enantioselective reduction of the acyl furans and the stereocontrol in the formation of the anomeric tert-butyl carbonates. Thus acyl furan 157 can be transformed, in a stereocontrolled manner in $\alpha-\mathrm{L}, \beta-\mathrm{L}, \alpha-\mathrm{D}$ or $\beta-\mathrm{D}$ tert-butyl carbonates 155 and 164, respectively (Scheme 40) [155]. These derivatives were used in the preparation of a collection of 11 methymycin analogues (179) by stereoselective glycosylation of 10-deoxymethylnolide 177 followed by synthetic manipulations of the ensuing pyranones 178 (Scheme 41).

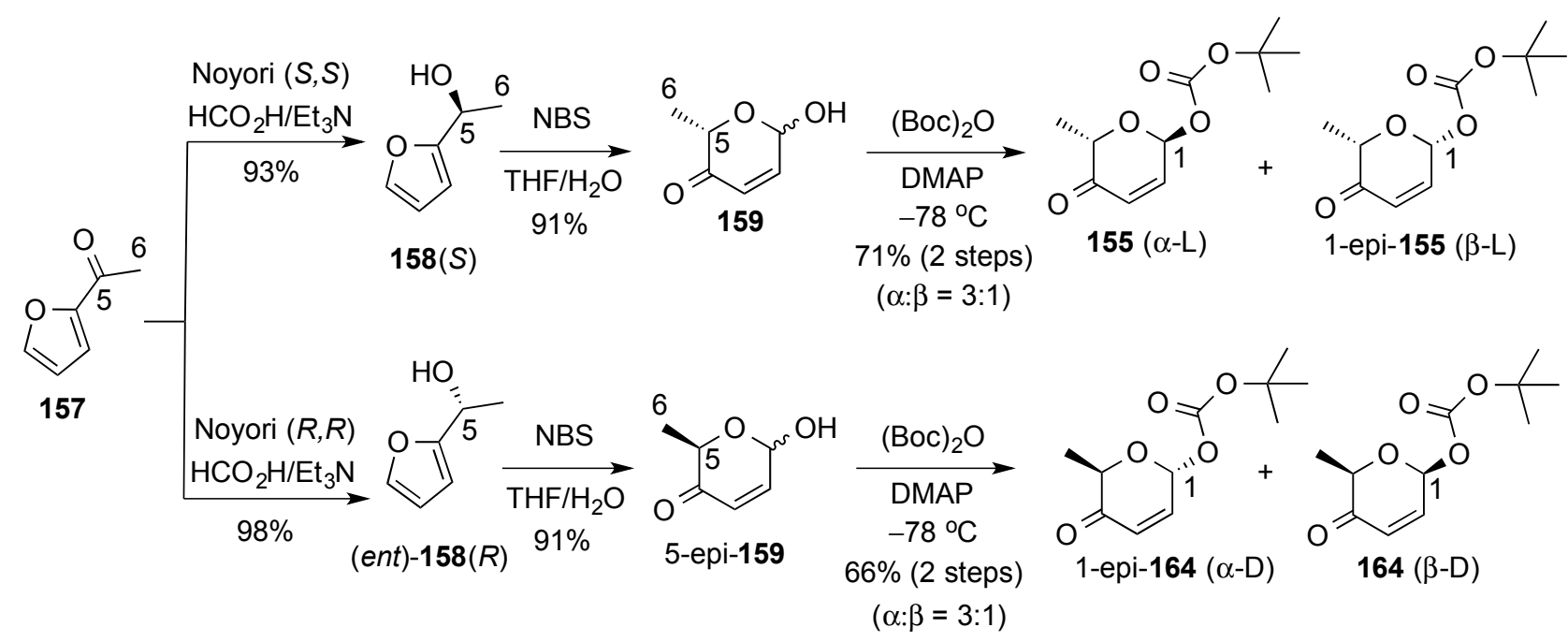

Scheme 40. O'Doherty's enantio- and stereo-divergent approach to $D / L$ and $\alpha / \beta$-pyranones 155 and 164.

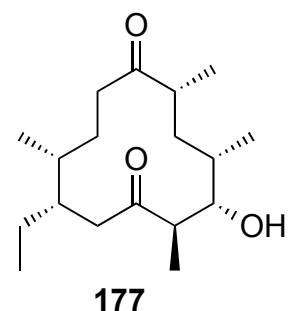

177

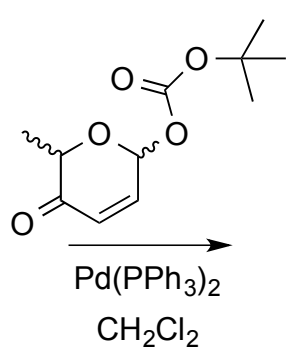

$\mathrm{CH}_{2} \mathrm{Cl}_{2}$

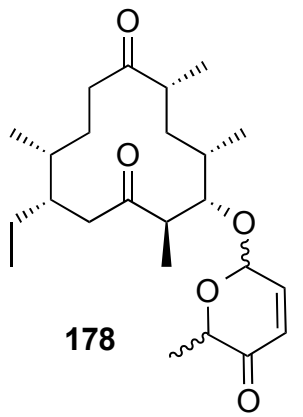
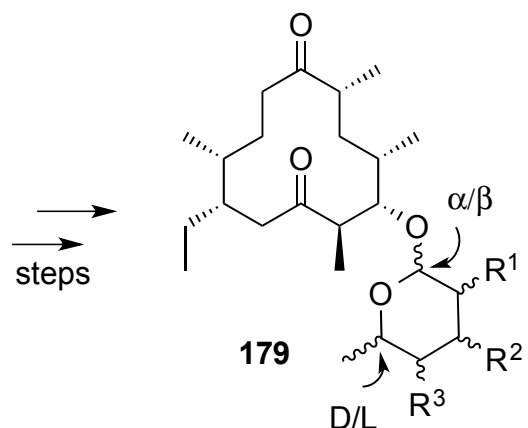

Scheme 41. Enantio- and stereo-divergent synthesis of glycosylated methymycin analogues, 179.

Related chemistry was also used in the preparation of the $\alpha$-L-aculose, $\alpha$-L-rhodinose, and $\beta$-D-olivose trisaccharide-component of PI-080 [156].

Pyranones with oxygen substituents at the primary position, e.g., 148c, 185, as precursors of 6-hydroxy pyranoses, can analogously be prepared in either enantiomeric form (D/L) from oxygenated acyl furan 180 by way of enantioselective Noyori reduction (181, 182), and Achmatowitcz ring-expansion (Scheme 42) [157]. A combination of D- and L-pyranones were used by O'Doherty in the de novo asymmetric synthesis of all-D, all-L, and D-/L- oligosaccharides (see Scheme 10, Section 3.1) [48]. 


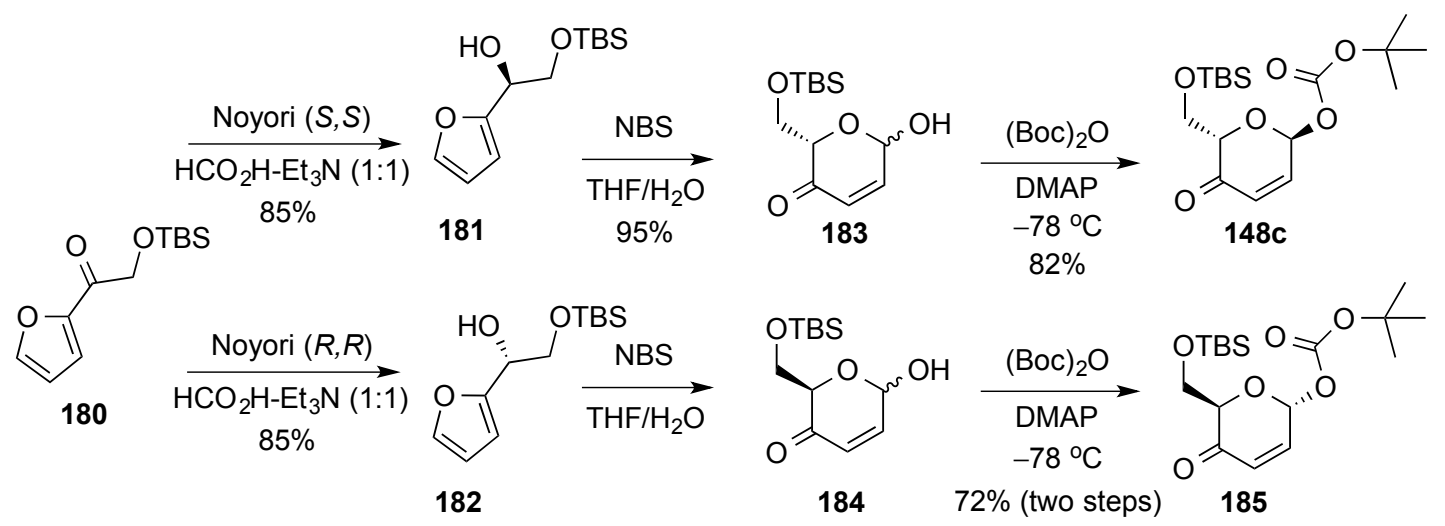

Scheme 42. Enantiodivergent synthesis of pyranones, $\mathbf{1 4 8 c}$ and $\mathbf{1 8 5}$ from acyl furan $\mathbf{1 8 0 .}$

Pyranone 185 has also been used in the preparation of the glycosylated tyrosine portion of mannopetimycin-E, 186 (Scheme 43) [158,159].<smiles>COC(=O)[C@H](Cc1ccc(O[C@H]2OC(CO)[C@H](O[C@H]3OC(CO)[C@@H](NC(=O)CC(C)C)[C@H](O)[C@H]3O)[C@H](O)[C@H]2O)cc1)NC(=O)O</smiles>

186

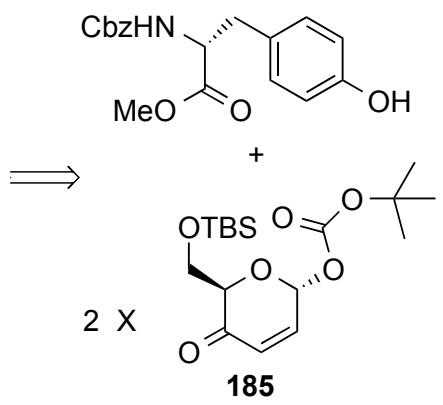

185

Scheme 43. O'Doherty's retrosynthesis of the disaccharide portion of mannopetimycin-E $\mathbf{1 8 6}$.

Very recently, Liu and co-workers have reported the stereoselective palladium-catalyzed $N$-glycosylation of $\alpha$-picoloyl 2,3-unsaturated hexopyranosides, e.g., 187, leading to $N$-heterocyclic glycosides 188 (Scheme 44) [160]. The method, initially developed and optimized on 3-picoloyl glucals, was compatible with a variety of protecting groups on the glycosyl donor.<smiles>CC(=O)OC1C=CC(OC(=O)c2ccccn2)OC1CO</smiles>

187

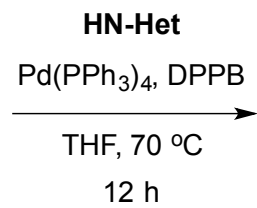

$12 \mathrm{~h}$

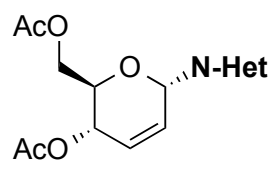

188<smiles>CC(=O)OCC1OC(N2C(=O)C(=O)c3ccccc32)C=C[C@H]1OC(C)=O</smiles>

(43\%)<smiles>CC(=O)OCC1OC(N2C(=O)C(=O)c3cc(C)ccc32)C=CC1[O+]=O</smiles>

(41\%)<smiles>CC(=O)OCC1OC(n2ccnc2-c2ccccc2)C=CC1OC(C)=O</smiles>

(47\%)<smiles>CC(=O)OCC1OC(N2C(=O)c3ccccc3C2=O)C=C[C@H]1OC(C)=O</smiles>

(83\%)

Scheme 44. Liu and co-workers' stereoselective palladium-catalyzed $N$-glycosylation.

Based on their results, the authors were able to propose a reaction mechanism that is outlined in Scheme 45. The pathway involved simultaneous initial palladium coordination to the double bond 
and to the nitrogen of the pycoloyl group at the $\alpha$-face of the sugar to generate intermediate 189 . Subsequent cleavage of the picoloyl acid species yielded the $\pi$-allyl system depicted as 190. Finally, coordination of the $N$-nucleophile to the palladium released the picoloyl acid and provided intermediate 191, where an intramolecular nucleophilic addition takes place to yield $N$-heterocyclic glycosides 188.

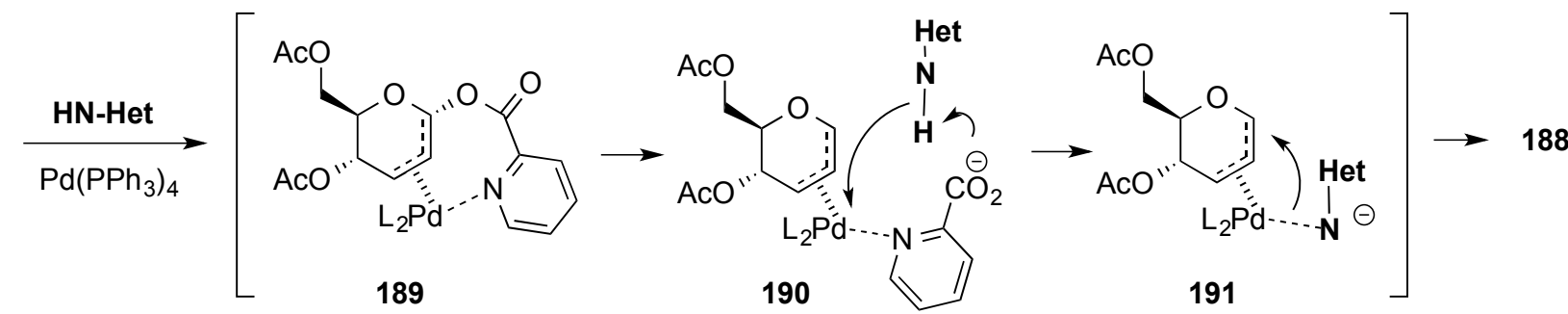

Scheme 45. Proposed reaction mechanism for the synthesis of $N$-glycosides 188.

\subsubsection{Lewis-Acid Mediated Glycosylation of 2,3-Unsaturated Glycosyl Acetates}

Toshima and co-workers reported on the chemoselective assembly of differently substituted 2,3-unsaturated pyranoses [161,162]. Thus, 2,3-unsaturated-4-keto glycosyl acetates, e.g., 193, were found to display lower reactivity than 2,3-unsaturated-4-hydroxy glycosyl acetates, e.g., 192, in the presence of Lewis acids, and could therefore be used as glycosyl acceptors with the latter acting as glycosyl donors. An implementation of their strategy is outlined in Scheme 46. Accordingly, 4-keto derivative 193 was chemoselectively glycosylated with 192 by use of TMSOTf in $\mathrm{CH}_{2} \mathrm{Cl}_{2}$ at $-78{ }^{\circ} \mathrm{C}$, to give disaccharide 194 in fairly good yield. Subsequently, the ensuing 4-keto derivative 194 was able to act as a glycosyl donor and was used to glycosylate methyl glucoside 195, in toluene at higher temperature, to yield trisaccharide 196 (TMSOTf, $-40{ }^{\circ} \mathrm{C}$ ). The observed $\alpha / \beta$ anomeric selectivity was high and in agreement with literature precedents favoring the $\alpha$-anomer in each case.

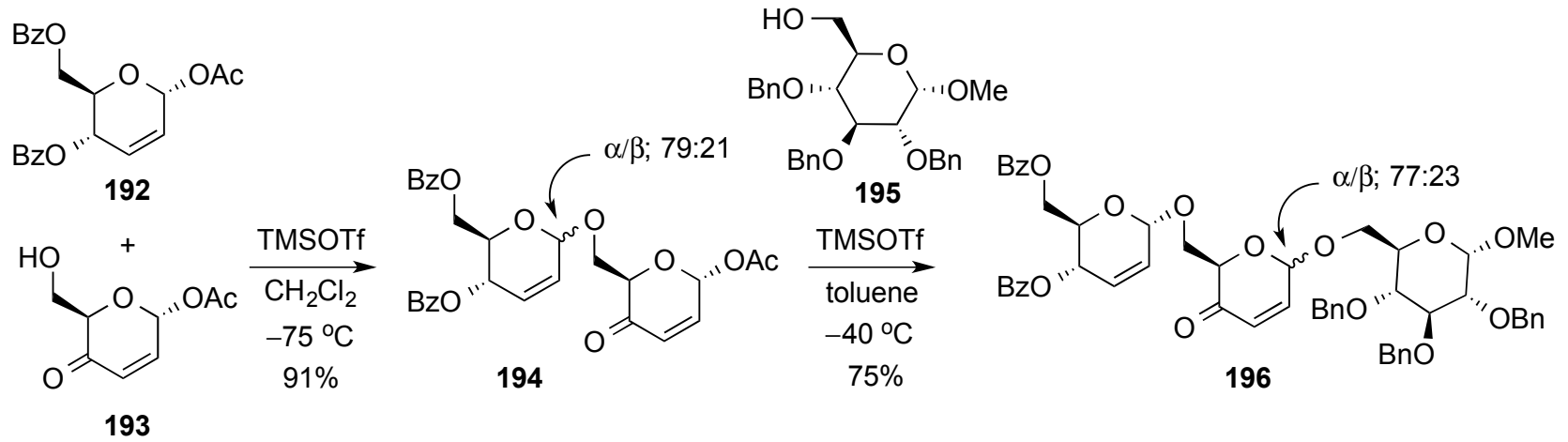

Scheme 46. Toshima's chemoselective glycosylation strategy to trisaccharide 196.

\subsubsection{Halonium Ion-Mediated Glycosylation of 2,3-Unsaturated Allyl Glycosides}

Taneja and co-workers recently described the remarkable stereoselective $\alpha$-glycosylation of 2,3-unsaturated allyl glycosides mediated by NBS in the presence of catalytic $\mathrm{Zn}(\mathrm{OTf})_{2}$ [163]. The method was applied to the glycosylation of a variety of alcohols with erythro- and threo-2,3-unsaturated 
allyl glycosides 197 and 198, respectively (Scheme 47). Protecting groups such as acetonide, nitro, or esters proved to be compatible with the reaction conditions.

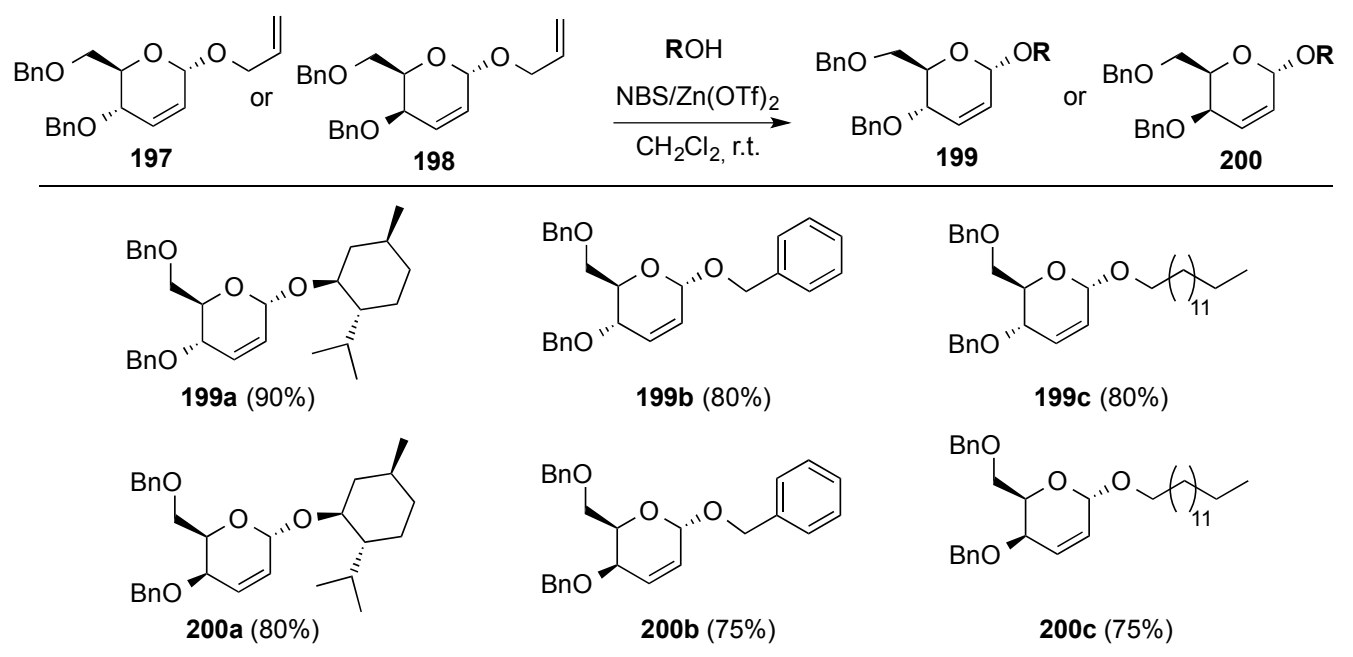

Scheme 47. Taneja's stereoselective $\alpha$-glycosylation with allyl glycosides 197 and 198.

\subsection{Use of 2,3-Unsaturated Hexopyranoses as Chiral Complex Ligands}

Carbohydrates have been long used as stereodifferentiating agents [164]. In this context, Boysen and co-workers reported on a phosphinite hybrid ligand 201, based in a 2,3-unsaturated pyranoside [165]. Accordingly, phosphinite 201, readily prepared by reaction of the corresponding unsaturated alcohol with diphenyl chlorophosphine ( $\mathrm{PPh}_{2} \mathrm{Cl}, \mathrm{Et}_{3} \mathrm{~N}, \mathrm{THF}, 70 \%$ yield), was employed in the rhodium-catalyzed 1,4-addition of boronic acids to unsaturated ketones and lactones 202. The ensuing products, 203, were obtained with high yields and excellent stereoselectivity when cyclic substrates were involved (Scheme 48). In a recent remarkable development, Boysen and co-workers reported that isomeric erythro-, i.e., 201, and threo-, i.e., 204, phosphinites, behaved as pseudo-enantiomeric olefin ligands in $\mathrm{Rh}(\mathrm{I})$-catalyzed 1,4-additions of aryl and alkenylboronic acids to achiral enones [166]. They also extended the reaction to a variety of alkenyl and aryl boronic acids.

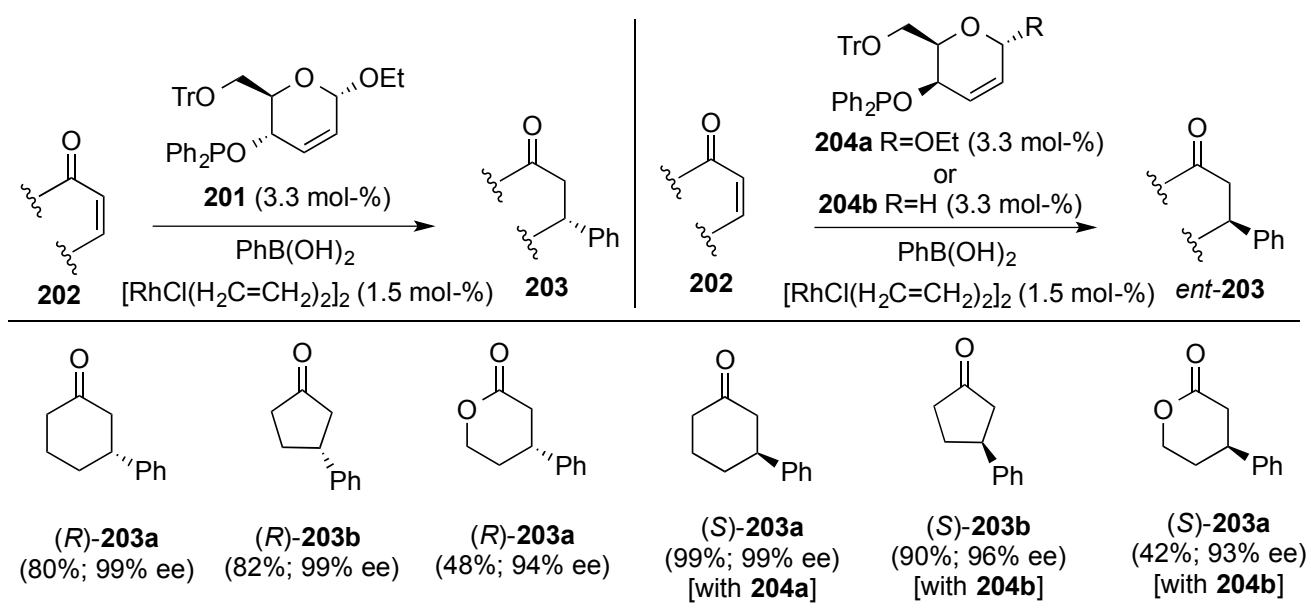

Scheme 48. Boysen's pair of pseudoenantiomeric carbohydrate derived phophinites 201 and 204, in rhodium catalyzed asymmetric 1,4-addition of phenylboronic acid to unsaturated enones and enoates. 


\subsection{Miscellaneous}

A series of synthetic transformations of de novo hex-2,3-enopyranose derivatives, e.g., 155, into a variety of monosaccharide and deoxy-monosaccharide derivatives have been described by O'Doherty's group [167]. These transformations make imaginative use of addition, oxidation, and substitution reactions performed on hex-2,3-enopyranoses and 3,4-unsaturated pyranoses, e.g., 207, the latter readily available from the former by Wharton rearrangement (Scheme 49) [168]. Accordingly, Boc-pyranone 155 was converted by way of stereoselective $\operatorname{Pd}(0)$ glycosylation into $\alpha$-benzyl derivative 205, whose epoxidation under basic conditions led stereoselectively to epoxy ketone 206 [169]. Wharton rearrangement of the latter then provided benzyl hex-3,4-enopyranoside 207.

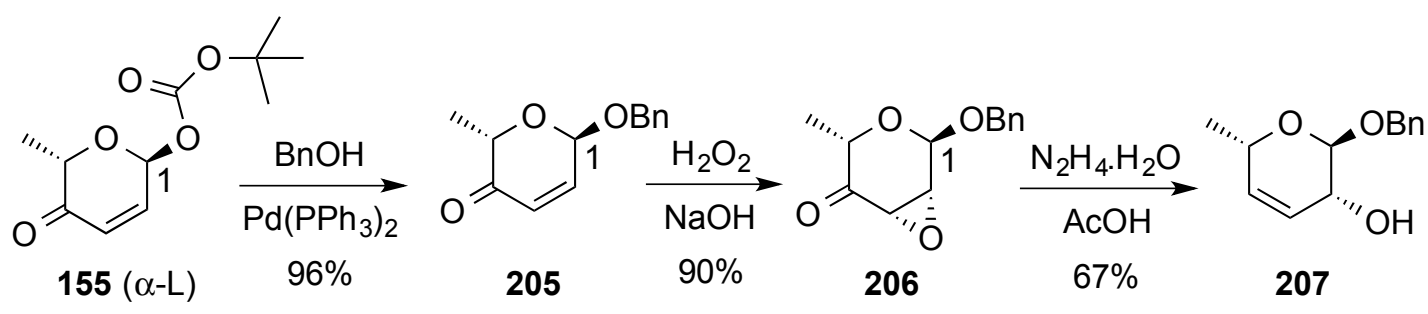

Scheme 49. Wharton rearrangement of pyranone 205 to hex-3,4-enopyranoside 207.

A synthetic route to $\alpha$-ascariloside 209, was devised by regio- and stereoselective reaction of $\mathbf{2 0 7}$ with $N$-iodosuccinimide (NIS) in acetic acid followed by $\mathrm{LiAlH}_{4}$ reduction of the ensuing $\beta$-acetoxy iodide 208 (Scheme 50) [169,170]. An approach to benzyl $\alpha$-fucoside (211) from 207 was implemented via osmylation of $\mathbf{2 1 0}$ (2-epi-207, prepared by oxidation/reduction of 207) (Scheme 50). It was observed that osmylation of $\mathbf{2 1 0}$ leading to fucose monosaccharides (211) was better carried out on 2-silyl derivative 210b, which produced a 7:1 diastereomeric mixture favoring $\mathbf{2 1 1 b}$ [211b/212b 7:1)]. Conversely, osmylation of $\mathbf{2 1 0 a}$ led to diastereomeric 212a as the major isomer [211a/212a 1:4)] [169].<smiles>C[C@H]1C=C[C@@H](O)[C@H](OCc2ccccc2)O1</smiles>

207

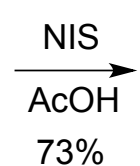

$73 \%$

i) $\mathrm{MnO}_{2}(76 \%)$

ii) $\mathrm{NaBH}_{4} / \mathrm{CeCl}_{3}(100 \%)$<smiles>[R]O[C@H]1O[C@H](C)C=C[C@H]1OCc1ccccc1</smiles>

210

a $\mathrm{R}=\mathrm{H}$

b $R=T B S$<smiles>[R]O[C@@H]1[C@H](Cc2ccccc2)O[C@H](O)[C@H](O)[C@@H]1O</smiles>

211

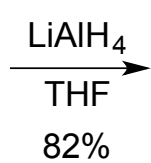

208<smiles>C[C@H]1O[C@H](Cc2ccccc2)[C@H](O)C[C@H]1O</smiles>

209<smiles>[R]O[C@@H]1[C@H](Cc2ccccc2)O[C@H](C)[C@@H](O)[C@@H]1O</smiles>

212

$$
\begin{array}{lll}
\text { a } R=H & 1: 4 & (80 \%) \\
\text { b } R=\text { TBS } & 7: 1 & (95 \%)
\end{array}
$$

Scheme 50. Synthetic transformations of hex-3,4-enopyranoside 207 leading to $\alpha$-ascariloside 209 and $\alpha$-fucosides 211. 


\section{Conclusions}

Hex-2,3-enopyranosides continue to be important intermediates currently used in a variety of synthetic transformations. They are readily available by Ferrier rearrangement of commercially available glycals, although more recently the de novo approach to pyranones, and thence hex-2,3-enopyranosides, has positioned itself as reliable synthetic alternative for their preparation. The latter approach has the advantage of providing access to enantiomeric hex-2,3-enopyranoside pairs. The use of 2,3-unsaturated pyranosides in glycosylation has grown exponentially during the last decade, more than likely because of the success on the stereoselective $\operatorname{Pd}(0)$-mediated glycosyl coupling of $\alpha$ - and $\beta$ - pyranones.

\section{Acknowledgments}

The authors wish to thank the Ministerio de Ciencia e Innovación for its financial support through grant CTQ2012-32114.

\section{Author Contributions}

AMG and JCL planned and supervised the review. AMG, FL, SM, and JCL participated in the writing of the manuscript.

\section{Conflicts of Interest}

The authors declare no conflict of interest.

\section{References and Notes}

1. Ferrier, R.J.; Zubkov, O.A. Transformation of glycals into 2,3-unsaturated glycosyl derivatives. Org. React. 2003, 62, 569-736.

2. Ferrier, R.J.; Hoberg, J.O. Synthesis and reactions of unsaturated sugars. Adv. Carbohydr. Chem. Biochem. 2003, 58, 55-119.

3. Fischer, E. Concerning new reduction products of dextrose, glucal and hydro-glucal. Chem. Ber. 1914, 47, 196-210.

4. Bergmann, M. The pseudo-glucal and dihydro-pseudoglucal. Liebigs Ann. Chem. 1925, 443, 223-242.

5. Ferrier, R.J.; Prasad, N. Unsaturated carbohydrates. IX. Synthesis of 2,3-dideoxy- $\alpha$-D-erythrohex-2-enopyranosides from tri-O-acetyl-D-glucal. J. Chem. Soc. C 1969, 570-575.

6. Ferrier, R.J. Unsaturated sugars. Adv. Carbohydr. Chem. Biochem. 1969, 24, 199-266.

7. Ferrier, R.J. Unsaturated sugars. In The Carbohydrates; Pigman, W., Horton, D., Eds.; Academic Press: New York, NY, USA, 1980; Volume 1B, p. 843.

8. Fraser-Reid, B. Some progeny of 2,3-unsaturated sugars-They little resemble grandfather glucose: Twenty years later. Acc. Chem. Res. 1996, 29, 57-66.

9. Fraser-Reid, B. Some progeny of 2,3-unsaturated sugars-They little resemble grandfather glucose: Ten years later. Acc. Chem. Res. 1985, 18, 347-354. 
10. Fraser-Reid, B. Some progeny of 2,3-unsaturated sugars-They little resemble grandfather glucose. Acc. Chem. Res. 1975, 8, 192-201.

11. The chemistry of 2,3-unsaturated derivatives on pyranoid systems had been periodically reviewed In Carbohydrate Chemistry, Specialist Periodical Reports; Royal Society of Chemistry: Cambridge, UK, 2003; from Volume 1, 1968, to Volume 34.

12. Fraser-Reid, B.; López, J.C. Unsaturated sugars: A rich platform for methodological and synthetic studies. Curr. Org. Chem. 2009, 13, 532-553.

13. Gómez, A.M.; Lobo, F.; Uriel, C.; López, J.C. Recent developments in the Ferrier rearrangement. Eur. J. Org. Chem. 2013, 2013, 7221-7262.

14. Tipson, R.S.; Cohen, A. Action of zinc dust and sodium iodide in $N, N$-dimethylformamide on contiguous, secondary sulfonyloxy groups: A simple method for introducing nonterminal unsaturation. Carbohydr. Res. 1965, 1, 338-340.

15. Fraser-Reid, B.; Boctor, B. Some aspects of the formation of hex-2-enopyranosides from methyl 2,3-di-O-methanesu1 fonyl- $\alpha$-D-glucopysanosides. Can. J. Chem. 1969, 47, 393-401.

16. Achmatowicz, O.; Bielski, R. Stereoselective total synthesis of methyl $\alpha-\mathrm{D}$ and a-L-glucopyranosides. Carbohydr. Res. 1977, 55, 165-176.

17. Achmatowicz, O.; Bukowski, B.; Szechner, B.; Zwierzchowska, Z.; Zamojski, A. Synthesis of methyl 2,3-dideoxy-DL-alk-2-enopyranosides from furan compounds. A general approach to the total synthesis of monosaccharides. Tetrahedron 1971, 27, 1973-1996.

18. Shimshock, S.J.; Waltermire, R.E.; DeShong, P. A total synthesis of ( \pm )-Tirandamycin B. J. Am. Chem. Soc. 1991, 113, 8791-8796.

19. Adger, B.M.; Barrett, C.; Brennan, J.; McKervey, M.A.; Murray, R.W. Oxidation of furans with dimethyldioxirane. J. Chem. Soc. Chem. Commun. 1991, 1553-1554.

20. Guo, H.; O’Doherty, G.A. De novo asymmetric synthesis of Daumone via a palladium-catalyzed glycosylation. Org. Lett. 2005, 7, 3921-3924.

21. Georgiadis, M.P.; Couladouros, E.A. Products from furans. 4. Selective oxidation of 2-furfuryl alcohol derivatives, in the presence of aryl thioethers, with $N$-bromosuccinimide (NBS). A new procedure for the preparation of 2H-pyran-3(6H)-ones. J. Org. Chem. 1986, 51, 2725-2727.

22. Kusakabe, M.; Kitano, Y.; Kobayashi, Y.; Sato, F. Preparation of optically active 2-furylcarbinols by dinetic resolution using the Sharpless reagent and their application in organic synthesis. J. Org. Chem. 1989, 54, 2085-2091.

23. Wahlen, J.; Moens, B.; de Vos, D.E.; Alsters, P.L.; Jacobs, P.A. Titanium silicalite 1 (TS-1) catalyzed oxidative transformations of furan derivatives with hydrogen peroxide. Adv. Synth. Catal. 2004, 346, 333-338.

24. Taniguchi, T.; Nakamura, K.; Ogasawara, K. Non-carbohydrate route to levoglucosenone and its enantiomer employing asymmetric dihydroxylation. Synlett 1996, 971-972.

25. Taniguchi, T.; Ohnishi, H.; Ogasawara, K. An expedient preparation of chiral building blocks having levoglucosenone chromophore: A new enantiocontrolled route to $(-)-\beta$-multistriatin and (+)-exo-brevicomin. Chem. Commun. 1996, 1477-1478.

26. Harris, J.M.; Keranen, M.D.; O’Doherty, G.A. Syntheses of D- and L-mannose, gulose, and talose via diastereoselective and enantioselective dihydroxylation reactions. J. Org. Chem. 1999, 64, 2982-2983. 
27. Deagostino, A.; Prandi, C.; Zavattaro, C.; Venturello, P. Functionalized 1-alkoxy-1,3-dienes: Their preparation and applications in synthetic organic chemistry. Eur. J. Org. Chem. 2006, 2463-2483.

28. Eftekhari-Sis, B.; Zirak, M. Chemistsry of $\alpha$-oxoesters: A powerful tool for the synthesis of heterocycles. Chem. Rev. 2015, 115, 151-264.

29. Frihen, T.G.; Bols, M.; Pedersen, C.M. Synthesis of L-hexoses. Chem. Rev. 2015, doi:10.1021/acs.chemrev.5b00104.

30. Bednarski, M.; Danishefsky, S. Interactivity of chiral catalysts and chiral auxiliaries in the cycloaddition of activated dienes with aldehydes: A synthesis of L-glucose. J. Am. Chem. Soc. 1986, 108, 7060-7067.

31. For a recent example of Danishefsky's diene in the preparation of carbohydrate mimetics, see: Burland, P.A.; Coisson, D.; Osborn, H.M.I. Rapid synthesis of carbohydrate derivatives, including mimetics of $C$-linked disaccharides and $C$-linked aza disaccharides, using the hetero-Diels-Alder reaction. J. Org. Chem. 2010, 75, 7210-7218.

32. Angerbauer, R.; Schmidt, R.R. Short synthesis of racemic uronic acids and 2,3-anhydrouronic acids. Carbohydr. Res. 1981, 89, 159-162.

33. Bataille, C.; Begin, G.; Guillam, A.; Lemiegre, L.; Lys, C.; Maddaluno, J.; Toupet, L. Thermal/hyperbaric heterocycloaddition of 1,4-dialkoxy-1,3-dienes: The de novo (E,Z) way to sugars. J. Org. Chem. 2002, 67, 8054-8062.

34. Wan, Q.; Lubineau, A.; Guillot, R.; Scherrmann, M.-C. Synthesis of $C$-disaccharides via a hetero-Diels-Alder reaction and further stereocontrolled transformations. Carbohydr. Res. 2008, 343, 1754-1765.

35. Castagnolo, D.; Botta, L.; Botta, M. One-pot multicomponent synthesis of 2,3-dihydropyrans: New access to furanose-pyranose 1,3-C-C-linked-disaccharides. Tetrahedron Lett. 2009, 50, 1526-1528.

36. Lopez, J.C.; Plumet, J. Metathesis reactions of carbohydrates: Recent highlights in alkyne metathesis. Eur. J. Org. Chem. 2011, 2011, 1803-1825.

37. Aljarilla, A.; Lopez, J.C.; Plumet, J. Metathesis reactions of carbohydrates: Recent highlights in cross-metathesis. Eur. J. Org. Chem. 2010, 2010, 6123-6143.

38. Crimmins, M.T.; King, B.W.; Zuercher, W.J.; Choy, A.L. An efficient, general asymmetric synthesis of carbocyclic nucleosides: Application of an asymmetric aldol/ring-closing metathesis strategy. J. Org. Chem. 2000, 65, 8499-8509.

39. Guaragna, A.; D’Alonzo, D.; Paolella, C.; Napolitano, C.; Palumbo, G. Highly stereoselective de novo synthesis of L-hexoses. J. Org. Chem. 2010, 75, 3558-3568.

40. Guaragna, A.; Napolitano, C.; D’Alonzo, D.; Pedatella, S.; Palumbo, G. A versatile route to L-hexoses: Synthesis of L-mannose and -altrose. Org. Lett. 2006, 8, 4863-4866.

41. Guppi, S.R.; Zhou, M.; O’Doherty, G.A. De novo asymmetric synthesis of homoadenosine via a palladium-catalyzed $N$-glycosylation. Org. Lett. 2006, 8, 293-296.

42. Groebke, K.; Hunziker, J.; Fraser, W.; Peng, L.; Diederichsen, U.; Zimmermann, K.; Holzner, A.; Leumann, C.; Eschenmoser, A. Why pentose- and not hexose-nucleic acids? Purine-purine pairing in homo-DNA: Guanine, isoguanine, 2,6-diaminopurine, and xanthine. Helv. Chim. Acta 1998, 81, 375-474. 
43. Hooper, I.R. The naturally occurring aminoglycoside antibiotics. In Aminoglycoside Antibiotics; Hooper, I.R., Umezawa, H., Eds.; Springer-Verlag: New York, NY, USA, 1982.

44. Zhang, G.; Shi, L.; Liu, Q.; Wang, J.; Li, L.; Liu, X. A divergent strategy for constructing a sugar library containing 2,6-dideoxy sugars and uncommon sugars with 4-substitution. Tetrahedron 2007, 63, 9705-9711.

45. Haukaas, M.H.; O’Doherty, G.A. Enantioselective synthesis of 2-deoxy- and 2,3-dideoxyhexoses. Org. Lett. 2002, 4, 1771-1774.

46. Murphy, P.V.; O’Brien, J.L.; Smith, A.B., III. Stereospecific synthesis of $\beta$-D-allopyranosides by dihydroxylation of $\beta$-D-erythro-2,3-dideoxyhex-2-enopyranosides. Carbohydr. Res. 2001, 334, 327-335.

47. Ellis, D.; Norman, S.E.; Osborn, H.M.I. Synthesis of S-linked carbohydrate analogues via a Ferrier reaction. Tetrahedron 2008, 64, 2832-2854.

48. Babu, R.S.; Chen, Q.; Kang, S.-W.; Zhou, M.; O’Doherty, G.A. De novo asymmetric synthesis of all-D-, all-L-, and D-/L oligosaccharides using atom-less protecting groups. J. Am. Chem. Soc. 2012, 134, 11952-11955.

49. Ferrier, R.J.; Prasad, N. Unsaturated carbohydrates. Part X. Epoxidations and hydroxylations of 2,3-dideoxy- $\alpha$-D-hex-2-enopyranosides. The four methyl 4,6-di- $O$-acetyl-2,3-anhydro- $\alpha$-Dhexopyranosides. J. Chem. Soc. C 1969, 575-580.

50. Angyal, S.J. Ring-opening of anhydrosugars of the ethylene oxide type. Chem. Ind. 1954, 1230-1231.

51. Joly, J.-P.; Roze, F.; Banas, S.; Quilès, F. Synthesis and Raman spectra of 3-deoxy- $\alpha$-L-rhamnosides as model sugars of the Ascaris egg shell. Tetrahedron Lett. 2010, 51, 3236-3241.

52. Sakakibara, T.; Yamada, M.; Sudoh, R. Phase transfer catalyzed reactions. II. Reactions of methyl 3-deoxy-3-nitro- $\beta$-D-hexopyranosides with active methylene compounds. J. Org. Chem. 1976, 41, 736-737.

53. Sakakibara, T.; Sudoh, R. Phase transfer catalyzed reactions. I. Highly stereoselective formation of the thermodynamically less stable manno isomers from nitro sugars with active methylene compounds. J. Org. Chem. 1975, 40, 2823-2825.

54. Sakakibara, T.; Tokuda, K.; Hayakawa, T.; Seta, A. Michael reactions on conformationally flexible methyl 3-C-nitro-hex-2-enopyranoside derivatives. Carbohydr. Res. 2000, 327, 489-496.

55. Nakagawa, T.; Sakakibara, S. Kumazawa. Tetrahedron Lett. 1970, 11, 1645-1648.

56. Rajaebalee, F.J.-M. Synthèse de dérivés amines du méthyl-3-desoxy-3-nitro- $\alpha$ - et B-D-glucopyranoside. Carbohydr. Res. 1973, 26, 219-224.

57. Sakakibara, T.; Sudoh, R. Stereochemistry of nucleophilic addition reactions. 2. Kinetically controlled reaction of methyl 4,6-O-benzylidene-2,3-dideoxy-3-nitro- $\beta$-D-erythro-hex-2enopyranoside with hydrogen cyanide. Important role of electrostatic interaction. J. Org. Chem. 1977, 42, 1746-1750.

58. Sakakibara, T.; Sudoh, R. Stereoselective synthesis of the thermodynamically less stable manno isomers from a nitro sugar. Carbohydr. Res. 1976, 50, 191-196.

59. Jegou, E.; Cleophax, J.; Leboul, J.; Gero, S.D. A facile synthesis of derivatives of lividosamine, a component of lividomycin B. Carbohydr. Res. 1975, 45, 323-326. 
60. Apostolopoulos, C.D.; Couladouros, E.A.; Georgiadis, M.P. Synthesis of sugar-amino acid conjugates via 2,3-dideoxy-hex-2-enopyranos-4-uloses. Liebigs Ann. Chem. 1994, 781-784.

61. Couladouros, E.A.; Constantinou- Kokotou, V.; Georgiadis, M.P.; Kokotos, G. A synthesis in one reaction vessel of 2,4-diamino sugar precursors from 2,3-dideoxy-2-enopyranos-4-uloses. Carbohydr. Res. 1994, 254, 317-324.

62. Sanki, A.K.; Bhattacharya, R.; Atta, A.K.; Suresh, C.G.; Pathak, T. Diastereoselective addition of planar $N$-heterocycles to vinyl sulfone-modified carbohydrates: A new route to isonucleosides. Tetrahedron 2008, 64, 10406-10416.

63. Bhattacharya, R.; Kesarwani, M.K.; Manna, C.; Ganguly, B.; Suresh, C.G.; Pathak, T. An experimental and theoretical study on the remarkable influence of protecting groups on the selectivity of addition of amines to vinyl sulfone-modified hex-2-enopyranosides. J. Org. Chem. 2010, 75, 303-314.

64. Ravindran, B.; Sakthivel, K.; Suresh, C.G.; Pathak, T. Diastereoselective addition of amines to vinyl sulfone modified carbohydrates: A highly flexible methodology for the synthesis of new classes of deoxyaminosugars. J. Org. Chem. 2000, 65, 2637-2641.

65. Sanki, A.K.; Suresh, C.G.; Falgune, U.D.; Pathak, T. Anomeric configuration-directed diastereoselective $\mathrm{C}-\mathrm{C}$ bond formation in vinyl sulfone-modified carbohydrates: A general route to branched-chain sugars. Org. Lett. 2003, 5, 1285-1288.

66. Bhattacharya, R.R.; Atta, A.K.; Dey, D.; Pathak, T. Densely functionalized chiral pyrroles from endocyclic, exocyclic, and acyclic vinyl sulfone-modified carbohydrates. J. Org. Chem. 2009, 74, 669-674.

67. Mukherjee, A.; Jayaraman, N. Facial selectivities in the nucleophilic additions of 2,3-unsaturated 3-arylsulfinyl pyranosides. Carbohydr. Res. 2013, 380, 51-58.

68. Ding, F.; Cai, S.; William, R.; Liu, X.-W. Pathways leading to 3-amino- and 3-nitro-2,3-dideoxy sugars: Strategies and synthesis. RSC Adv. 2013, 3, 13594-13621.

69. Pauls, H.W.; Fraser-Reid, B. An efficient synthesis of ristosamine utilizing the allylic hydroxyl of an hex-2-enopyranoside. J. Org. Chem. 1983, 48, 1392-1393.

70. Bongini, A.; Cardillo, G.; Orena, M.; Sandri, S.; Tomasini, C. A regio- and stereoselective synthesis of methyl $\alpha$-L-ristosaminide hydrochloride. Tetrahedron 1983, 39, 3801-3806.

71. Pauls, H.W.; Fraser-Reid, B. Stereocontrolled routes to cis-hydroxyamino sugars, part VII: Synthesis of daunosamine and ristosamine. Carbohydr. Res. 1986, 150, 111-119.

72. Nicolaou, K.C.; Baran, P.S.; Zhong, Y.-L.; Barluenga, S.; Hunt, K.W.; Kranich, R.; Vega, J.A. Iodine(V) reagents in organic synthesis. Part 3. New routes to heterocyclic compounds via $o$-iodoxybenzoic acid-mediated cyclizations: Generality, scope, and mechanism. J. Am. Chem. Soc. 2002, 124, 2233-2244.

73. Doi, T.; Shibata, K.; Kinbara, S.; Takahashi, T. A divergent route to 3-amino-2,3,6-trideoxysugars including branched sugar: Synthesis of vancosamine, daunosamine, saccharosamine, and ristosamine. Chem. Lett. 2007, 36, 1372-1373.

74. Dorgan, B.J.; Jackson, R.F.W. Synthesis of $C$-linked glycosyl amino acid derivatives using organozinc reagents. Synlett 1996, 859-861.

75. Chapleur, Y.; Grapsas, Y. Stereospecific formation of carbon-carbon bonds in ethyl 4,6-di-Oacetyl-2,3-dideoxy- $\alpha$-D-ribo-hex-3-enopyranoside. Carbohydr. Res. 1985, 141, 153-158. 
76. Danishefsky, S.J.; Armistead, D.M.; Wincott, F.E.; Selnick, H.G.; Hungate, R. The total synthesis of Avermectin A1a. J. Am. Chem. Soc. 1989, 111, 2967-2980.

77. Valverde, S.; Bernabé, M.; Garcia-Ochoa, S.; Gómez, A.M. Regio- and stereochemistry of cross coupling of organocopper reagents with allyl ethers: Effect of the leaving group. J. Org. Chem. 1990, 55, 2294-2298.

78. Valverde, S.; Bernabe, M.; Gomez, A.M.; Puebla, P. Cross coupling reactions of 2-(allyloxy(thio))benzothiazoles with organocopper reagents in dihydropyranoid systems. Mechanistic implications of the substrate and the reagent: Regio- and stereocontrolled access to branched-chain sugars. J. Org. Chem. 1992, 57, 4546-4550.

79. Hyodo, T.; Katayama, Y.; Kobayashi, Y. Allylic substitution on the pyran ring. Tetrahedron Lett. 2009, 50, 3547-3549.

80. RajanBabu, T.V. Pd(0)-Catalyzed C-glycosylation: A facile alkylation of trifluoroacetylglucal. J. Org. Chem. 1985, 50, 3642-3644.

81. Dunkerton, L.V.; Euske, J.M.; Serino, A.J. Palladium(0)-assisted synthesis of $C$-glycopyranosyl compounds. Carbohydr. Res. 1987, 171, 89-107.

82. Curran, D.P.; Suh, Y.-G. Selective mono-Claisen rearrangement of carbohydrate glycals. A chemical consequence of the vinylogous anomeric effect. Carbohydr. Res. 1987, 171, 161-191.

83. Brakta, M.; Lhoste, P.; Sinou, D. Palladium(0)-based approach to functionalized C-glycopyranosides. J. Org. Chem. 1989, 54, 1890-1896.

84. Engelbrecht, G.J.; Holzapfel, C.W. Palladium-catalyzed reactions of unsaturated carbohydrates-A route to $C$-glycosides. Heterocycles 1991, 32, 1267-1272.

85. Brescia, M.-R.; Shimshock, Y.C.; DeShong, P. Regioselectivity in the palladium-catalyzed addition of carbon nucleophiles to dihydropyran derivatives. J. Org. Chem. 1997, 62, 1257-1263.

86. Yeager, A.R.; Min, G.K.; Porco, J.A.; Schaus, S.E. Exploring skeletal diversity via ring contraction of glycal-derived scaffolds. Org. Lett. 2006, 8, 5065-5068.

87. Mendonça, F.J.B.; dos Anjos, J.V.; Sinou, D.; de Melo, S.J.; Srivastava, R.M. Palladium-catalyzed alkynylation (Sonogashira coupling) at C-5 of the uracil moiety in modified unsaturated pyranosyl nucleosides. Synthesis 2007, 1890-1897.

88. De Oliveira, R.N.; Cottier, L.; Sinou, D.; Srivastava, R.M. Stereocontrolled palladium(0)-catalyzed preparation of unsaturated azidosugars: An easy access to 2- and 4-aminoglycosides. Tetrahedron 2005, 61, 8271-8281.

89. Mrozowski, R.M.; Sansuaky, Z.M.; Vemula, R.; Wu, B.; Zhang, Q.; Lannigan, D.A.; O'Doherty, G.A. De novo synthesis and biological evaluation of C6"-substituted C4" amide analogues of SL0101. Org. Lett. 2014, 16, 5996-5999.

90. Ferrier, R.J.; Vethaviyasar, N. Unsaturated carbohydrates. XVII. Synthesis of branched-chain sugar derivatives by the Claisen rearrangement. J. Chem. Soc. Perkin Trans 1 1973, 1791-1793.

91. Krohn, K.; Flörke, U.; Gehle, D. Highly deoxygenated sugars. I. C2-Branched glucose derivatives and carbon linked deoxygenated disaccharides. J. Carbohydr. Chem. 2002, 21, 431-443.

92. Montero, A.; Mann, E.; Herradon, B. Preparation of sugar amino acids by Claisen-Johnson rearrangement: Synthesis and incorporation into enkephalin analogues. Eur. J. Org. Chem. 2004, 2004, 3063-3073. 
93. Kriek, N.M.A.J.; van der Hout, E.; Kelly, P.; van Meijgaarden, K.E.; Geluk, A.; Ottenhoff, T.H.M.; van der Marel, G.A.; Overhand, M.; van Boom, J.H.; Valentijn, A.R.P.M.; et al. Synthesis of novel tetrahydropyran-based dipeptide isosters by Overman rearrangement of 2,3-didehydroglycosides. Eur. J. Org. Chem. 2003, 2003, 2418-2427.

94. Montero, A.; Mann, E.; Herradon, B. The Overman rearrangement in carbohydrate chemistry: Stereoselective synthesis of functionalized 3-amino-3,6-dihydro- $2 \mathrm{H}$-pyrans and incorporation in peptide derivatives. Tetrahedron Lett. 2005, 46, 401-405.

95. Montero, A.; Benito, E.; Herradon, B. Synthesis and applications of a chiral-oxygenated 3-chloro-3,6-dihydro-2H-pyran obtained under Overman rearrangement conditions. Tetrahedron Lett. 2010, 51, 277-280.

96. Saquib, M.; Husain, I.; Sharma, S.; Yadav, G.; Singh, V.K.; Sharma, S.K.; Shah, P.; Siddiqi, M.I.; Kumar, B.; Lal, J.; et al. 2,3-Dideoxy hex-2-enopyranosid-4-uloses as promising new anti-tubercular agents: Design, synthesis, biological evaluation and SAR studies. Eur. J. Med. Chem. 2011, 2217-2223.

97. Saquib, M.; Husain, I.; Kumar, B.; Shaw, A.K. Facile synthesis of enantiomerically pure 2- and 2,3-disubstituted furans catalysed by mixed Lewis acids: An easy route to 3-iodofurans and 3-(hydroxymethyl)furans. Chem. Eur. J. 2009, 15, 6041-6049.

98. Bashiardes, G.; Cano, C.; Mauzé, C. Regio- and enantioselective synthesis of novel functionalized pyrano-pyrrolidines by 1,3-dipolar cycloaddition of carbohydrates. Synlett 2005, 587-590.

99. Filho, J.R.F.; Srivastava, R.M.; da Silva, W.J.P.; Cottier, L.; Sinou, D. Synthesis of new branched-chain amino sugars. Carbohydr. Res. 2003, 338, 673-680.

100. By, K.; Kelly, P.A.; Kurth, M.J.; Olmstead, M.M.; Nantz, M.H. Synthesis of a C(4)-C(9) eleutheside template from D-glucal. Tetrahedron 2001, 57, 1183-1187.

101. Taillefumier, C.; Chapleur, Y. Enantiomerically pure decalinic structures from carbohydrates using intramolecular Diels-Alder and Ferrier carbocyclization. Can. J. Chem. 2000, 78, 708-722.

102. Gomez, A.M.; Lopez, J.C.; Fraser-Reid, B. Stereoselective synthesis of ethyl (Z)- and (E)-octa2,6-dienopyranosideuronates from ethyl 2,3-dideoxy- $\alpha$-D-erythro-hex-2-eno-pyranoside. Synlett 1993, 557-560.

103. Corey, E.J.; Kim, C.U. Improved synthetic routes to prostaglandins utilizing sulfide-mediated oxidation of primary and aecondary alcohols. J. Org. Chem. 1973, 38, 1233-1234.

104. Fraser-Reid, B.; Molino, B.F.; Magdzinski, L.; Mootoo, D.R. Tripyranoside precursors for ansamycins. Pyranosidic homologation. 6. J. Org. Chem. 1987, 52, 4505-4511.

105. Fraser-Reid, B.; Magdzinski, L.; Molino, B.F. New strategy for carbohydrate-based syntheses of multichiral arrays: Pyranosidic homologation. 3. J. Am. Chem. Soc. 1984, 106, 731-734.

106. Molino, B.F.; Magdzinski, L.; Fraser-Reid, B. Pyranosidic homologation: Part I: Extending the carbohydrate template via C6 and C4. Tetrahedron Lett. 1983, 24, 5819-5822.

107. Gomez, A.M.; Lopez de Uralde, B.; Valverde, S.; Lopez, J.C. A novel entry to naturally occurring 5-alkenyl $\alpha, \beta$-unsaturated $\delta$-lactones from d-glucose: Syntheses of $(+)$-acetylphomalactone and (+)-asperlin. Chem. Commun. 1997, 1647-1648.

108. Mieczkowski, J.; Jurczak, J.; Chmielewski, M.; Zamojski, A. The synthesis of 2,3-dideoxyhex-2enono-1.5 lactones. Carbohydr. Res. 1977, 56, 180-182. 
109. A related oxidation from hex-2-enopyranosides via photolysis of the corresponding 2-oxopropyl glycosides was described. Bernasconi, C.; Cottier, L.; Descotes, G.; Remy, G. Dégradations ménagées des sucres. Synthese par voie photochimique d'aldonolactones-1,5 a partir d'oxo-2 propylglycosides. Bull. Soc. Chim. Fr. 1979, 332-336.

110. Jarglis, P.; Lichtenthaler, F.W. Boron trifluoride-catalyzed oxidation of glycal esters: An effective and mild method for their conversion into $\alpha, \beta$-unsaturated lactones. Tetrahedron Lett. 1982, 23, 3781-3784.

111. Rollin, P.; Sinaÿ, P. A convenient, one-step oxidation of glycals to lactones using pyridinium chlorochromate. Carbohydr. Res. 1981, 98, 139-142.

112. Panfil, I.; Chmielewski, M. Cycloaddition of nitrones and $\alpha, \beta$-unsaturated sugar lactones. Tetrahedron 1985, 41, 4713-4716.

113. Panfil, I.; Belzecki, C.; Urbanczyk-Lipkowska, Z.; Chmielewski, M. 1,3-Dipolar cycloaddition of nitrones to sugar enlactones. Tetrahedron 1991, 47, 10087-10094.

114. Jurczak, M.; Rabiczko, J.; Socha, D.; Chmielewski, M.; Cardona, F.; Goti, A.; Brandi, A. Diastereoselection in 1,3-dipolar cycloadditions of a chiral cyclic nitrone to $\alpha, \beta$-unsaturated -lactones. Tetrahedron Asymmetry 2000, 11, 2015-2022.

115. Socha, D.; Jurczak, M.; Frelek, J.; Klimek, A.; Rabiczko, J.; Urbanczyk-Lipkowska, Z.; Suwinska, K.; Chmielewski, M.; Cardona, F.; Goti, A.; et al. 1,3-Dipolar cycloaddition of a nitrone derived from $(S)$-malic acid to $\alpha, \beta$-unsaturated $\delta$-lactones. Tetrahedron Asymm. 2001, 12 , 3163-3172.

116. Lopez, J.C.; Lameignere, E.; Lukacs, G. Stereospecificity in Diels-Alder reactions of dienes and dienophiles derived from methyl 4,6-O-benzylidene- $\alpha$-D-glucopyranoside. J. Chem. Soc. Chem. Commun. 1988, 706-707.

117. Lopez, J.C.; Lameignere, E.; Lukacs, G. Straightforward route to 2- and 3-formyl hex-1- and -2-enopyranosides and their highly stereoselective hetero Diels-Alder reaction with ethyl vinyl ether. J. Chem. Soc. Chem. Commun. 1988, 514-515.

118. Lipshutz, B.H.; Nguyen, S.L.; Elworthy, T.R. Preparation and Diels-Alder reactions of a pyranoid vinyl glycal: Model studies for anthraquinone aglycone and carbohydrate syntheses. Tetrahedron 1988, 44, 3355-3364.

119. Stecko, S.; Jurczak, M.; Panfil, I.; Furman, B.; Grzeszczyk, B.; Chmielewski, M. Synthesis of iminosugars via 1,3-dipolar cyloaddition reactions of nitrones to $\alpha, \beta$-unsaturated sugar aldonolactones. Comptes Rendus Chim. 2011, 14, 102-125.

120. Pasniczek, K.; Socha, D.; Jurczak, M.; Frelek, J.; Suszczynska, A.; Urbanczyk-Lipkowska, Z.; Chmielewski, M. Double asymmetric induction in 1,3-dipolar cycloaddition of nitrones to 2,3-unsaturated sugar 1,5-lactones. J. Carbohydr. Chem. 2003, 22, 613-629.

121. Pasniczek, K.; Socha, D.; Jurczak, M.; Solecka, J.; Chmielewski, M. Synthesis of 8-homocastanospermine. Can. J. Chem. 2006, 84, 534-539.

122. Socha, D.; Pasniczek, K.; Jurczak, M.; Solecka, J.; Chmielewski, M. Synthesis of 1-homoaustraline. Carbohydr. Res. 2006, 341, 2005-2011.

123. Pasniczek, K.; Jurczak, M.; Urbanczyk-Lipkowska, Z.; Chmielewski, M. Synthesis of 2,3-dihydroxy-1-epilupinine. J. Carbohydr. Chem. 2007, 26, 195-211. 
124. Panfil, I.; Urbanczyk-Lipkowska, Z.; Chmielewski, M. Synthesis of iminosugars from $\alpha, \beta$-unsaturated lactones and $N$-benzyl nitrone. Pol. J. Chem. 2005, 79, 239-249.

125. Panfil, I.; Solecka, J.; Chmielewski, M. Synthesis of (-)-isofagomine. J. Carbohydr. Chem. 2006, 25, 673-684.

126. Stecko, S.; Pasniczek, K.; Jurczak, M.; Urbanczyk-Lipkowska, Z.; Chmielewski, M. Kinetic and thermodynamic aspects in the 1,3-dipolar cycloaddition of five-membered cyclic nitrones to $\alpha, \beta$-unsaturated $\gamma$ - and $\delta$-lactones. Tetrahedron Asymmetry 2007, 18, 1085-1093.

127. Testero, S.A.; Spanevello, R.A. Enantiospecific approach toward pentalenolactone. Org. Lett. 2006, 8, 3793-3796.

128. Lopez, J.C.; Lameignere, E.; Burnouf, C.; Laborde, M.A.; Ghini, A.; Olesker, A.; Lukacs, G. Efficient routes to pyranosidic homologated conjugated enals and dienes from monosaccharides. Tetrahedron 1993, 49, 7701-7722.

129. Testero, S.A.; Spanevello, R.A. The first example of a highly non-symmetric ozonolysis of a sugar derived norbornene system. ARKIVOC 2003, 220-226, doi:10.3998/ark.5550190.0004.a22.

130. Mangione, M.I.; Testero, S.A.; Suarez, A.G.; Spanevello, R.A.; Tuchages, J.-P. Synthesis and spectroscopic NMR studies of a highly stable cross-ozonide product derived from a carbohydrate system. Tetrahedron Assymetry 2006, 17, 1780-1785.

131. Testero, S.A.; Mangione, M.I.; Poeylaut-Palena, A.; González-Sierra, M.; Spanevello, R.A. Unsymmetrical ozonolysis of carbohydrate derived norbornene systems. Tetrahedron 2007, 63, 11410-11420.

132. Mangione, M.I.; Suarez, A.G.; Spanevello, R.A. Synthesis of methyl 4,6-O-benzylidene-2,3dideoxy-2-C-formyl- $\alpha$-D-erythro-hex-2-enopyranoside. Carbohydr. Res. 2005, 340, 149-153.

133. Mangione, M.I.; Sarotti, A.M.; Suarez, A.G.; Spanevello, R.A. Experimental and theoretical study of a Diels-Alder reaction between a sugar-derived nitroalkene and cyclopentadiene. Carbohydr. Res. 2011, 346, 460-464.

134. For an excellent review on transition metal-catalyzed glycosylations, see McKay, M.J.; Nguyen, H.M. Recent advances in transition metal-catalyzed glycosylation. ACS Catal. 2012, 2, 1563-1595.

135. Van der Deen, H.; van Oeveren, A.; Kellogg, R.M.; Feringa, B.L. Palladium catalyzed stereospecific allylic substitution of 5-acetoxy-2 $(5 H)$-furanone and 6-acetoxy- $2 H$-pyran3 $(6 H)$-one by alcohols. Tetrahedron Lett. 1999, 40, 1755-1758.

136. Comely, A.C.; Eelkema, R.; Minnaard, A.J.; Feringa, B.L. De novo asymmetric bio- and chemocatalytic synthesis of saccharides-Stereoselective formal $O$-glycoside bond formation using palladium catalysis. J. Am. Chem. Soc. 2003, 125, 8714-8715.

137. Babu, R.S.; O’Doherty, G.A. A palladium-catalyzed glycosylation reaction: The de novo synthesis of natural and unnatural glycosides. J. Am. Chem. Soc. 2003, 125, 12406-12407.

138. Kim, H.; Men, H.; Lee, C. Stereoselective palladium-catalyzed $O$-glycosylation using glycals. J. Am. Chem. Soc. 2004, 126, 1336-337.

139. Kim, H.; Lee, C. A mild and efficient method for the stereoselective formation of C-O bonds: Palladium-catlyzed allylic etherification using zinc(II) alkoxides. Org. Lett. 2002, 4, 4369-4371. 
140. Harris, J.M.; Keranen, M.D.; Nguyen, H.; Young, V.G.; O’Doherty, G.A. Syntheses of four D- and L-hexoses via diastereoselective and enantioselective dihydroxylation reactions. Carbohydr. Res. 2000, 328, 17-36.

141. Fujii, A.; Hashiguchi, S.; Uematsu, N.; Ikariya, T.; Noyori, R. Ruthenium(II)-catalyzed asymmetric transfer hydrogenation of ketones using a formic acid-triethylamine mixture. J. Am. Chem. Soc. 1996, 118, 2521-2522.

142. Babu, R.S.; Zhou, M.; O’Doherty, G.O. De novo synthesis of oligosaccharides using a palladium-catalyzed glycosylation reaction. J. Am. Chem. Soc. 2004, 126, 3428-3429.

143. Zhou, M.; O’Doherty, G.A. A stereoselective synthesis of digitoxin and digitoxigen mono- and bisdigitoxoside from digitoxigenin via a palladium-catalyzed glycosylation. Org. Lett. 2006, 8, 4339-4342.

144. Zhou, M.; O’Doherty, G.A. De Novo Approach to 2-Deoxy- $\beta$-glycosides: Asymmetric Syntheses of Digoxose and Digitoxin. J. Org. Chem. 2007, 72, 2485-2493.

145. Guo, H.; O’Doherty, G.A. De novo asymmetric synthesis of anthrax tetrasaccharide by a palladium-catlyzed glycosylation reaction. Angew. Chem. Int. Ed. Engl. 2007, 46, 5206-5208.

146. Guo, H.; O’Doherty, G.A. De novo asymmetric synthesis of anthrax tetrasaccharide and related tetrasaccharide. J. Org. Chem. 2008, 73, 5211-5220.

147. Wang, H.-Y.L.; Guo, H.; O’Doherty, G.A. De novo asymmetric synthesis of rhamno di- and tri-saccharides related to the anthrax tetrasaccharide. Tetrahedron 2013, 69, 3432-3436.

148. Zhou, M.; O'Doherty, G.A. De novo synthesis of the trisaccharide subunit of landomycins A and E. Org. Lett. 2008, 10, 2283-2286.

149. Wu, B.; Li, M.; O’Doherty, G.A. Synthesis of several cleistrioside and cleistetroside natural products via a divergent de novo asymmetric approach. Org. Lett. 2010, 12, 5466-5469.

150. Shan, M.; O’Doherty, G.A. De novo asymmetric syntheses of SL0101 and its analogues via a palladium-catalyzed glycosylation. Org. Lett. 2006, 8, 5149-5152.

151. Shan, M.; Sharif, E.U.; O'Doherty, G.A. Total synthesis of Jadomycin A and a carbasugar analogue of jadomycin B. Angew. Chem. Int. Ed. Engl. 2010, 49, 9492-9495.

152. Sharif, E.U.; O'Doherty, G.A. Biosynthesis and total synthesis studies on the jadomycin family of natural products. Eur. J. Org. Chem. 2012, 2095-2108.

153. Chen, Q.; Zhong, Y.; O’Doherty, G.A. Convergent de novo synthesis of vineomycinone $\mathrm{B}_{2}$ methyl ester. Chem. Commun. 2013, 49, 6806-6808.

154. Aljahdali, A.Z.; Shi, P.; Zhong, Y.; O’Doherty, G.A. De novo asymmetric synthesis of the pyranoses: From monosaccharides to oligosaccharides. Adv. Carbohydr. Chem. Biochem. 2013, 69, 55-123.

155. Borisova, S.A.; Guppi, S.R.; Kim, H.J.; Wu, B.; Penn, J.H.; Liu, H.-W.; O’Doherty, G.A. A de novo approach to the synthesis of glycosylated methymycin analogues with structural and stereochemical diversity. Org. Lett. 2010, 12, 5150-5153.

156. Yu, X.; O’Doherty, G.A. De novo asymmetric synthesis and biological evaluation of the trisaccharide portion of PI-080 and vineomycin B2. Org. Lett. 2008, 10, 4529-4532.

157. Li, M.; Scott, J.; O'Doherty, G.A. Synthesis of 7-oxa-phomopsolide E and its C-4 epimer. Tetrahedron Lett. 2004, 45, 1005-1009. 
158. Guppi, S.R.; O’Doherty, G.A. Synthesis of aza-analogues of the glycosylated tyrosine portion of mannopeptimycin-E. J. Org. Chem. 2007, 72, 4966-4969.

159. Babu, R.S.; Guppi, S.R.; O’Doherty, G.A. Synthetic studies toward mannopectimycin-E:

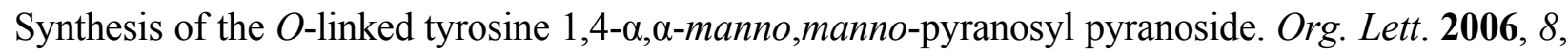
1605-1608.

160. Ji, L.; Xiang, S.-H.; Leng, W.-L.; Hoang, K.L. M.; Liu, X.-W. Palladium-catalyzed glycosylation: Novel synthetic approach to diverse $N$-heterocyclic glycosides. Org. Lett. 2015, 17, 1357-1360.

161. Kusumi, S.; Sasaki, K.; Wang, S.; Watanabe, T.; Takahashi, D.; Toshima, K. Effective and chemoselective glycosylations using 2,3-unsaturated sugars. Org. Biomol. Chem. 2010, 8, 3164-3178.

162. Kusumi, S.; Wang, S.; Watanabe, T.; Sasaki, K.; Takahashi, D.; Toshima, K. Chemoselective glycosylations using 2,3-unsaturated-4-keto glycosyl donors. Org. Biomol. Chem. 2010, 8, 988-990.

163. Kumar, B.; Aga, M.A.; Rouf, A.; Shah, B.A.; Taneja, S.C. 2,3-Unsaturated allyl glycosides as glycosyl donors for selective $\alpha$-glycosylation. J. Org. Chem. 2011, 76, 3506-3510.

164. Lehnert, T.; Ozuduru, G.; Grugel, H.; Albrecht, F.; Telligmann, S.M.; Boysen, M.M.K. More than just sweet-Sugar-derived stereodifferentiating agents for asymmetric synthesis. Synthesis 2011, 17, 2685-2708.

165. Minuth, T.; Boysen, M.M.K. Novel, efficient alkene-phosphinite hybrid ligand based on D-glucose. Org. Lett. 2009, 11, 4212-4215.

166. Grugel, H.; Albrecht, F.; Minuth, T.; Boysen, M.M.K. Efficient pseudo-enantiomeric carbohydrate olefin ligands. Org. Lett. 2012, 14, 3780-3783.

167. Cuccarese, M.F.; Wang, H.-Y.L.; O’Doherty, G.A. Application of the Wharton rearrangement for the de novo synthesis of pyranosides with ido, manno, and colito stereochemistry. Eur. J. Org. Chem. 2013, 3067-3075.

168. Wharton, P.; Bohlen, D. Hydrazine reduction of $\alpha, \beta$-epoxy ketones to allylic alcohols. J. Org. Chem. 2011, 76, 3506-3510.

169. Wang, H.-Y.L.; O’Doherty, G.A. De novo synthesis of deoxy sugar via a Wharton rearrangement. Chem. Commun. 2011, 47, 10251-10253.

170. An alternative approach to $\alpha$-ascarilose from keto-epoxide 201 had previously been described: Shan, M.; Xing, Y.; O’Doherty, G.A. De novo asymmetric synthesis of an a-6-deoxyaltropyranoside as well as its 2-/3-deoxy and 2,3-dideoxy congeners. J. Org. Chem. 2009, 74, 5961-5966.

(C) 2015 by the authors; licensee MDPI, Basel, Switzerland. This article is an open access article distributed under the terms and conditions of the Creative Commons Attribution license (http://creativecommons.org/licenses/by/4.0/). 